क्ष

\section{Determination of Coupled-Lattice Properties Using Turn-By-Turn Data}

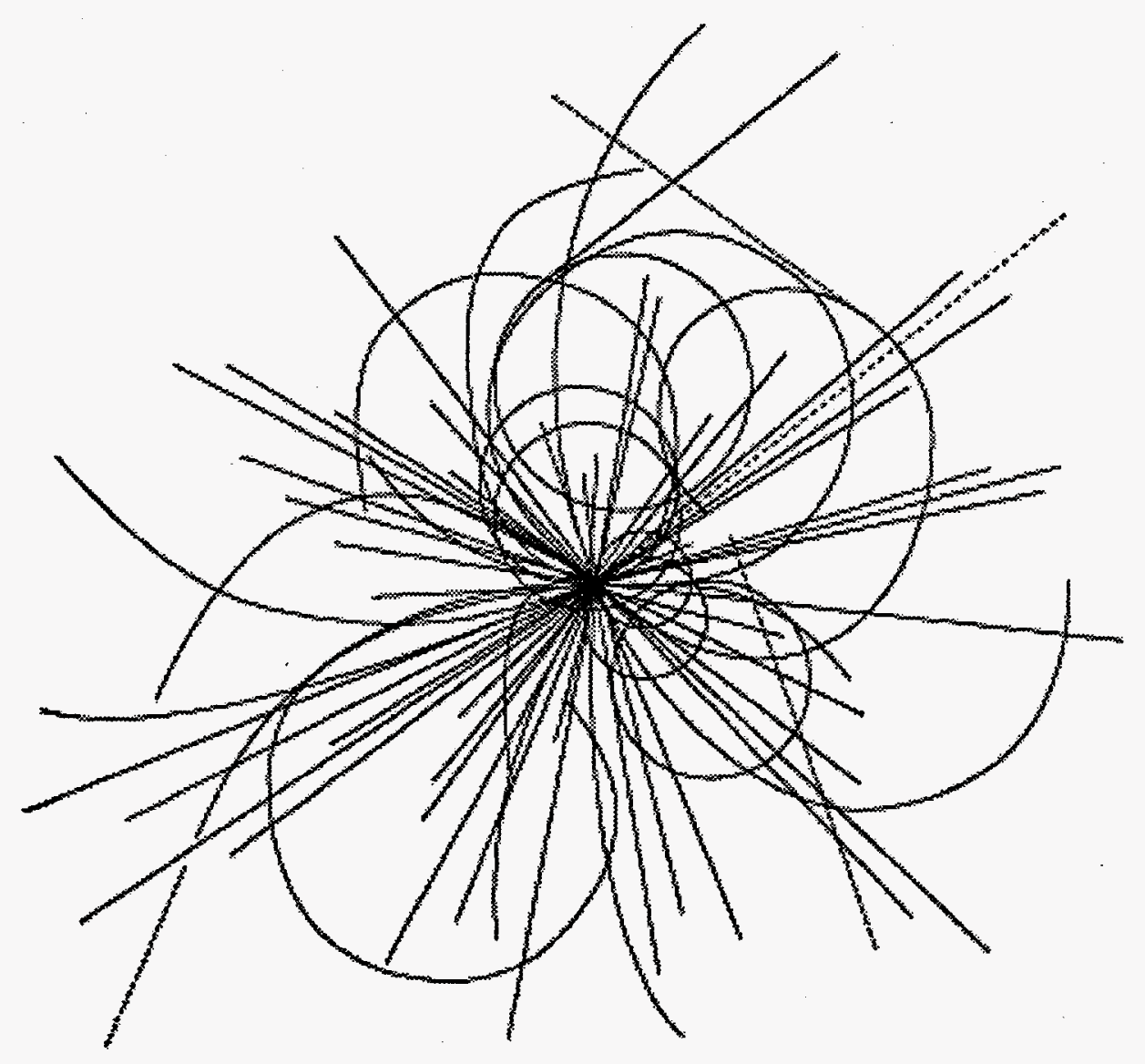

\section{Superconducting Super Collider Laboratory}

CONF-9210?7--15 SSCL-Preprint-181

December 1992

Distribution Category: 400

G. Bourianoff

S. Hunt

D. Mathieson

F. Pilat

R. Talman

G. Morpurgo 


\section{Disclaimer Notice}

This report was prepared as an account of work sponsored by an agency of the United States Govemment. Neither the United States Government of any agency thereot, nor any of their employees, makes any warranty, express or implied, or assumes any legal liability or responsibility for the accuracy, completeness, or uselulness of any information, apparatus, product, or process disclosed, or represents that its use would not infringe privately owned rights. Reference herein to any specific commercial product, process, or service by trade name, trademark, manufacturer, or otherwise, does not necessarily constitute or imply its endorsement, recommendation, or favoring by the Uniled States Government or any agency thereof. The views and opinions of authors expressed herein do not necessarily state or reflect those of the United States Govemment or any agency thereot.

Superconducting Super Collider Laboratory is an equal opportunity employer. 


\title{
Determination of Coupled-Lattice Properties Using Turn-By-Turn Data*
}

\author{
G. Bourianoff, S. Hunt, D. Mathieson, F. Pilat, R. Talman \\ Superconducting Super Collider Laboratory ${ }^{\dagger}$ \\ 2550 Beckleymeade Ave. \\ Dallas, TX 75237
}

G. Morpurgo

CERN

Geneva, Switzerland

December 1992

\section{DISCLAIMER}

\begin{abstract}
This report was prepared as an account of work sponsored by an agency of the United States Government. Neither the United States Government nor any agency thereof, nor any of their employees, makes any warranty, express or implied, or assumes any legal liability or responsibility for the accuracy, completeness, or usefulness of any information, apparatus, product, or process disclosed, or represents that its use would not infringe privately owned rights. Reference herein to any specific commercial product, process, or service by trade name, trademark, manufacturer, or otherwise does not necessarily constitute or imply its endorsement, recommendation, or favoring by the United States Government or any agency thereof. The views and opinions of authors expressed herein do not necessarily state or reflect those of the United States Government or any agency thereof.
\end{abstract}

*Presented at the Workshop on the Stability of Particle Motion in Storage Rings, Brookhaven National Laboratory, October 1992.

fOperated by the Universities Research Association, Inc., for the U.S. Department of Energy under Contract No. DE-AC35-89ER40486. 



\section{DISCLAIMER}

Portions of this document may be illegible in electronic image products. Images are produced from the best available original document. 


\title{
DETERMINATION OF COLPLED-LATTICE PROPERTIES USING TURN-BY-TURN DATA
}

\author{
G Bourianoff, S. Hunt, D. Mathieson, F. Pilat, R. Talman \\ SSC Laboratory, Dallas, TX, USA \\ G. Morpurgo \\ CERN, Geneva, Switzerland
}

\begin{abstract}
A formalism for extracting coupled betatron parameters from multiturn, shock excited, beam position monitor data is described. The most important results are nonperturbative in that they do not rely on the underlying ideal lattice model. Except for damping, which is assumed to be exponential and small enough to be removed empirically, the description is symplectic. As well as simplifying the description, this leads to self-consistency checks that are applied to the data. The most important of these is a "magic ratio" of Fourier coefficients that is required to be a lattice invariant, the same at every beam position monitor. All formulas are applied to both real and simulated data. The real data was acquired June, 1992 at LEP as part of deccoupling studies, using the LEP beam orbit measurement system. Simulated data, obtained by numerical tracking (TEAPOT) in the same (except for unknown errors) lattice, agrees well with real data when subjected to identical analysis. For both datasets, deviations between extracted and design parameters and deviations from self-consistency can be accounted for by noise and signal processing limitations. This investigation demonstrates that the LEP beam position system yields reliable local coupling measurements. It can be conservatively assumed that systems of similar design at the SSC and LHC will provide the measurements needed for local decoupling.
\end{abstract}




\section{Introduction}

The formulas in this report come from. and expand upon, those in a paper by Talman, ${ }^{[1]}$ which generalizes formulas of Courant and Snyder. ${ }^{\left[{ }^{[1}\right.}$ Except for rectifying a couple of unfortunate choices of symbols, or where new quantities are introduced, the notation is the same as in those papers. Formulas needed for this report are copied without proof. Also various explanations from those papers are assumed to be understood or are repeated only cursorily.

Only free oscillations following a transverse beam kick are analysed. Description of the equally important case of steady state response to a sinuisoidallyshaken beam would be essentially similar, though the likely simultaneous presence of both transient and steady state responses is likely to complicate that situation in practice. The analysis is heavily dependent on tune domain analysis, both for isolating eigenmotions and for noise suppression.

There is no discussion of methods that use adjacent BPM's to determine trajectory slopes, or of other methods that rely heavily on the ideal lattice model in the analysis. This also makes the formulas applicable to the analysis of feedback systems that sense beam properties at a single point. For the same reasons we defer multiplying the horizontal and vertical signals by factors $\sqrt{1 / \beta_{x}}$ and $\sqrt{1 / \beta_{y}}$ respectively, even though, to the extent these quantities are unperturbed, that "normalizes" the geometry appropriately, as will be shown. Some results can only be obtained by feeding in information from the lattice model, but they are inherently perturbative, and we judge it valuable to see how much can be extracted in a model-independent way. The data is analysed in terms of eigenmodes. The motion in one, nominally horizontal mode labeled $A$, wobbles around the horizontal $x$-plane. The nominally vertical mode is labeled $D$. (The letters $A$ and $D$ come from the Courant-Snyder ${ }^{[2]}$ notation for the block diagonal elements of the lattice transfer matrix.)

It is assumed that the BPM (beam position monitor) data acquisition system is perfect, so that digital values of the horizontal coordinate $x$ and the vertical coordinate $y$ correctly reflect the beam centroid every turn, for at least several hundred turns. Nonlinear amplitude response of the BPM's is assumed absent or compensated for and it is assumed that all BPM's have accurate calibrations that make the length scales equal at all lattice locations and in both planes.

The analysis assumes betatron amplitudes sufficiently small for the motion to be purely linear though, emphasizing the tune domain as it does, the method may be somewhat tolerant of nonlinearity. The lattice functions $\beta_{x}, \beta_{y}$, $\alpha_{x}$, and $\alpha_{y}$ of the (presumably decoupled) lattice are known and are available for use in perturbative calculations, but such use will be deferred as long as possible. 
Much of this report is devoted to deriving and using relationships among three "representations" of the motion:

- Coefficients extracted by Fourier analysis from the lists of displacements $(x, y)$ measured at all BPM's. There are six independent coefficients at each BPM. Directly derivable from these are

- transfer functions relating the $x$ and $y$ motions at each BPM, for each of two independent "modes". Each transfer function is parameterized by an amplitude ratio and a phase.

- Coordinates of successive turns at each BPM are related by the sixteen matrix elements of a once-around transfer matrix. Especially because only coordinates $x$ and $y$ are measured, while slopes $p$ and $q$ are not, not all matrix elements are uniquely derivable from the data.

In oversimplified terms, the two mode-tunes $\nu_{A}$ and $\nu_{D}$ are obtained by locating peaks in Fourier spectra, the two mode-emittances $\epsilon_{A}$ and $\epsilon_{D}$ are obtained from magnitudes of Fourier coefficients, and three of four off-diagonal transfer matrix elements are determined by ratios of Fourier coefficients. An important off-diagonal determinant (equivalent to the minimum tune split) constrains the Fourier coefficients but appears not to be derivable from turn-by-turn data at a single BPM in a model-independent way. As well as $\nu_{A}, \nu_{D}, \epsilon_{A}$, and $\epsilon_{D}$, the other parameters that are extracted in a model-independent way are $\beta_{A}, \beta_{D}$, one of the off-diagonal matrix elements, $R_{A 12}$, and the "magic ratio" $\mathcal{R}_{m}$ (which is equal to $\left.\epsilon_{D} / \epsilon_{A}\right)$. This ratio is deserving of the name magic because it is (or should be) a lattice invariant, even in the face of the nasty uninvited effects, coupling in the betatron motion and noise in the actual BPM system.

\section{Application of the Formulas to Realistic Data}

In this report, formulas useful for measuring lattice parameters are tested on two data sets. Each of these data sets consists of nominally horizontal free oscillations yielding measured $(x, y)$ pairs for 1024 turns at each of 504 BPM detectors of LEP. One data set, labeled 14_18, was acquired during a June, 1992 run on the nominally decoupled LEP accelerator. The other was obtained by TEAPOT $^{[3]}$ tracking for the same LEP lattice with the strengths of skew error elements having been increased until the coupling badness (defined below and in the accompanying report) was approximately equal to that of the actual data. The skew elements introduced to do this were random quad rolls (r.m.s. roll angle $=2 \mathrm{mr}$ ) and a (relatively unimportant) systematic skew quadrupole multipole in all dipoles ( $a_{1}=0.01$ "units". "units" refers to the SSC convention of parts per $10^{4}$ at $1 \mathrm{~cm}$.) For both real and simulated data, even though excitation of the 
"wrong-plane" eigenmotion was unintentional, there is enough signal to extract the most important parameters of both eigenmotions.

In almost all cases in the following the analyses of simulated data and actual data are completely parallel and remarkably similar. For that reason and to save space because there are a large number of figures, all plots are given in pairs, with simulated data on top and measured data on the bottom.

The first such pair, in Fig. 1, shows superimposed, Poincaré ( $x, y$ projection) plots at two adjacent BPM's. This particular pair of BPM's was chosen because of the near equality of their lattice function values: $\beta_{x}=59 \mathrm{~m}$ and $\beta_{y}=64 \mathrm{~m}$. These BPM's are symmetrically placed $\pm 14 \mathrm{~m}$ from an intersection region. Being equalbeta points, the transverse distributions can be regarded as "natural" without either axis having been magnified or demagnified by the lattice optics. Though the motion was intended to be purely horizontal and the lattice was "globally decoupled" (as will be reviewed below), it can be seen that vertical amplitudes are comparable to horizontal, and the orientation of the plane of oscillation can change rapidly. These deviations are great enough that a purely perturbative description of the cross-plane coupling is likely to be inadequate. With the much greater coupling strengths expected in the SSC or LHC (because of the much smaller magnet aperture and longer circumference) this will be all the more true for those accelerators. That is one reason the present non-perturbative description was developed. 

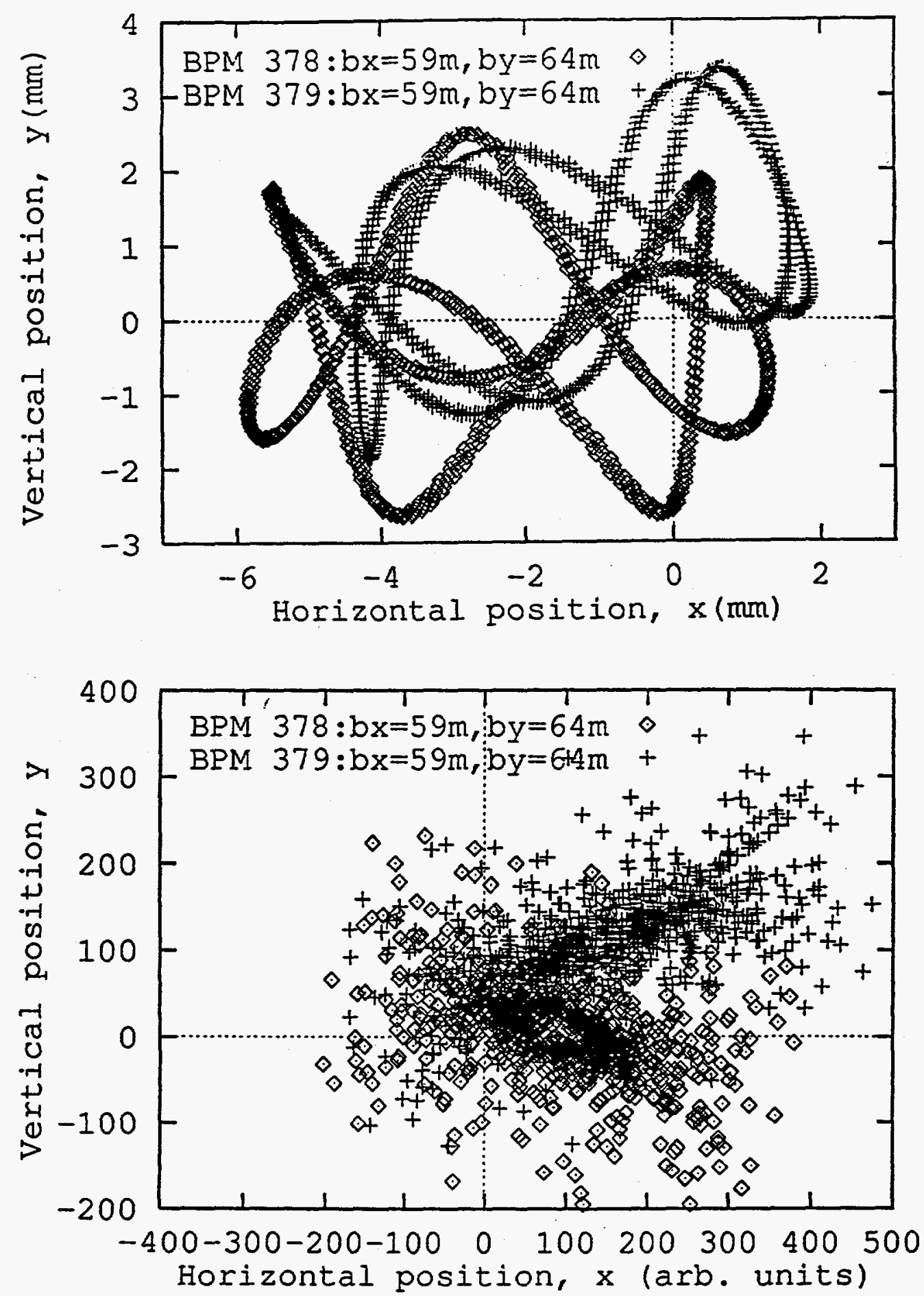

Figure 1. Two superimposed Poincaré plots ( $x, y$ projections) at adjacent BPM's for which $\beta_{x}$ and $\beta_{y}$ are roughly equal. This shows what would be observed on a (nondestructive) phosphor screen inserted at those points. Like all figures in this report the upper figure shows simulated results and the lower figure shows measured results. 
3. The Once-Around Transfer Matrix

The column vector of coordinates $x=(x, p, y, q)^{T}$ represents small transverse deviations from the reference orbit. Evolution of a vector $x$ from longitudinal coordinate $s_{0}$ to $s$ is described by a transfer matrix $M$,

$$
x(s)=M\left(s, s_{0}\right) x\left(s_{0}\right)
$$

A $4 \times 4$ transfer matrix $M$ is assumed to give an adequate description; effects of longitudinal motion are ignored. Using the matrix

$$
S=\left(\begin{array}{cccc}
0 & -1 & 0 & 0 \\
1 & 0 & 0 & 0 \\
0 & 0 & 0 & -1 \\
0 & 0 & 1 & 0
\end{array}\right),
$$

the symplectic condition $M$ must satisfy is

$$
M^{T} S M=S
$$

"Symplectic conjugation" of any matrix $A$ is defined by

$$
\bar{A}=-S A^{T} S
$$

For a $2 \times 2$ matrix with non-vanishing determinant

$$
\bar{A}=\overline{\left(\begin{array}{ll}
a & b \\
c & d
\end{array}\right)}=\left(\begin{array}{cc}
d & -b \\
-c & a
\end{array}\right)=A^{-1} \operatorname{det}|A|
$$

$2 \times 2$ elements of the partitioned $4 \times 4$ matrix $M$ and its symplectic conjugate are defined by

$$
M=\left(\begin{array}{cc}
A & B \\
C & D
\end{array}\right) ; \quad \bar{M}=\left(\begin{array}{cc}
\bar{A} & \bar{C} \\
\bar{B} & \bar{D}
\end{array}\right)
$$

Because $M$ is symplectic

$$
\bar{M}=M^{-1}
$$

The eigenvalues $\lambda$ of $M$ satisfy $\operatorname{det}|M-\lambda I|=0$. The combination

$$
M+\bar{M}=M+M^{-1}=\left(\begin{array}{cc}
A+\bar{A} & B+\bar{C} \\
C+\bar{B} & D+\bar{D}
\end{array}\right)=\left(\begin{array}{cc}
\operatorname{tr} A & \bar{E} \\
E & \operatorname{tr} D
\end{array}\right)
$$

turns out to have simpler properties than $M$, where the off-diagonal matrix $E$ 
and its determinant $\mathcal{E}$ are defined by

$$
E=C+\bar{B}=\left(\begin{array}{ll}
e & f \\
g & h
\end{array}\right) ; \quad \operatorname{det}|E|=e h-f g \equiv \mathcal{E},
$$

This matrix $M+M^{-1}$ exploits the fact that the eigenvalues of $M$ come in reciprocal pairs so that its eigenvalues are two doubly-degenerate real values, of the form $\Lambda=\lambda+\lambda^{-1}$. These sums are real even though the individual eigenvalues $\lambda$ are complex. The eigenvalue equation is

$$
\operatorname{det}\left|\begin{array}{cc}
(\operatorname{tr} A-\Lambda) I & \bar{E} \\
E & (\operatorname{tr} D-\Lambda) I
\end{array}\right|=\operatorname{tr} A \operatorname{tr} D-(\operatorname{tr} A+\operatorname{tr} D) \Lambda+\Lambda^{2}-\mathcal{E}=0
$$

whose solutions are

$$
\Lambda_{A, D}=(\operatorname{tr} A+\operatorname{tr} D) / 2 \pm \sqrt{(\operatorname{tr} A-\operatorname{tr} D)^{2} / 4+\mathcal{E}}
$$

where $A(D)$ goes with the $+(-) \operatorname{sign}$ if $\operatorname{tr} A-\operatorname{tr} D$ is positive and vice versa. This choice assures, for weak coupling, that $A$ will correspond to $x$ (this will be called the "nominally horizontal" eigenmotion) and $D$ will correspond to $y$ (the "nominally vertical" eigenmotion.) The eigenvalues satisfy simple equations

$$
\begin{aligned}
\Lambda_{A}+\Lambda_{D} & =\operatorname{tr} A+\operatorname{tr} D \\
\Lambda_{A} \Lambda_{D} & =\operatorname{tr} A \operatorname{tr} D-\mathcal{E} .
\end{aligned}
$$

In the physically important case, the magnitudes of the eigenvalues of $M$ do not exceed 1 , so that there are real angles $\mu_{A}=2 \pi \nu_{A}$ and $\mu_{D}=2 \pi \nu_{D}$ satisfying

$$
\begin{gathered}
\Lambda_{A} \equiv \lambda_{A}+1 / \lambda_{A}=\exp \left(i \mu_{A}\right)+\exp \left(-i \mu_{A}\right)=2 \cos \mu_{A} \\
\Lambda_{D} \equiv \lambda_{D}+1 / \lambda_{D}=\exp \left(i \mu_{D}\right)+\exp \left(-i \mu_{D}\right)=2 \cos \mu_{D}
\end{gathered}
$$

In the special uncoupled case, for which $B$ and $C$ vanish, these angles degenerate into the horizontal and vertical phase advances $\mu_{x}$ and $\mu_{y}$ which satisfy

$$
\Lambda_{A, D}=\operatorname{tr} A, D=2 \cos \mu_{x, y}=2 \cos \mu_{A, D}
$$

The determinant $\mathcal{E}$ has special significance since an accelerator is "globally decoupled" if and only if $\mathcal{E}=0$. This is achieved operationally by adjusting skew quad correction elements so that the two eigentunes can be made to coincide as 
nearly as possible when $(\operatorname{tr} A-\operatorname{tr} D)$ is scanned through zero using erect quads. That this will work can be seen by manipulating Eqs. (12) and (14) to get

$$
\left(\cos \mu_{A}-\cos \mu_{D}\right)^{2}=\frac{1}{4}(\operatorname{tr} A-\operatorname{tr} D)^{2}+\mathcal{E} .
$$

The left hand side is small close to either "sum" or "difference" resonances. The former case must be avoided since in that case it turns out that $\mathcal{E}<0$ and the motion is unstable; in the latter case $\mathcal{E}>0$ and the tune separation (or rather the squared difference of cosines) cannot be less than $\mathcal{E}$. The minimum tune separation is given by

$$
\left|\nu_{D}-\nu_{A}\right|_{\min }=\frac{\sqrt{\mathcal{E}}}{\pi\left(\sin \mu_{A}+\sin \mu_{D}\right)}
$$

The routine accelerator operation called global decoupling consists of adjusting skew quads (typically wired in two families) to minimize this minimum tune separation. This operation was performed on LEP just before the data set labeled 14-18 was acquired.

\section{Determination of the Eigenvectors}

To determine the eigenvectors of $M+M^{-1}$ it is useful to represent a displacement within the $x$ phase space by $\chi^{T}=(x, p)$ and similarly $\xi^{T}=(y, q)$. For eigenvalue $\Lambda$ it is easy to check that the vectors

$$
X=\left(\begin{array}{c}
\chi \\
\frac{E}{\Lambda-\operatorname{tr} D} \chi
\end{array}\right) ; \quad Y=\left(\begin{array}{c}
\frac{E}{\Lambda-\operatorname{tr} A} \xi \\
\xi
\end{array}\right)
$$

satisfy the equations

$$
\left(M+M^{-1}\right) X=\Lambda X ; \quad\left(M+M^{-1}\right) Y=\Lambda Y
$$

for either eigenvalue $\Lambda$ and arbitrary $\chi$ or $\xi$. By picking $\Lambda_{A}$ in defining $X$ and $\Lambda_{D}$ in defining $Y$, the nominally horizontal motion labelled $A$ is close to pure $x$ motion, and the nominally vertical motion is close to pure $y$ motion. Toward this end we define $2 \times 2$ matrices $R_{A}$ and $R_{D}$ by

$$
R_{A}=\frac{E}{\Lambda_{A}-\operatorname{tr} D} ; \quad R_{D}=\frac{\bar{E}}{\Lambda_{D}-\operatorname{tr} A}
$$


in terms of which independent basis vectors can be written as (proportional to)

$$
X=\left(\begin{array}{c}
\chi \\
R_{A} \chi
\end{array}\right) ; \quad Y=\left(\begin{array}{c}
-\bar{R}_{A} \xi \\
\xi
\end{array}\right)
$$

where a result that follows from Eq. (12),

$$
R_{D}=-\bar{R}_{A} .
$$

has been used.

\section{A Digression Concerning Data Processing Strategy}

The ideal goal of this analysis would be to extract all 16 elements of $M$ from the turn-by-turn data, but it will not be possible to do this completely. One reason for this is that only coordinates $(x, y)$ are measured; the slope variables $(p, q)$ are either unknown or must be inferred indirectly. Certain functions of the matrix elements, such as tunes, traces, or determinants, may be obtainable more directly than individual elements, but the introduction of such quantities at least temporarily increases the number of parameters to be determined. It is sensible to work to eliminate redundant variables, in order to identify a minimum set, and to develop optimal extraction procedures for those. In this spirit Eq. (21) has been used to eliminate $R_{D}$. It will be shown below that three, but not all four, of the elements of $R_{A}$ are directly measurable. The fact that, in preparation for taking the data the lattice was globally decoupled, implies that the determinant $\operatorname{det}\left|R_{A}\right|$ is expected to be small at every BPM location. But it would be "cheating" to exploit that in determining the fourth element of $R_{A}$; it would violate the spirit of this analysis. Rather we reserve this as a potential consistency check. Obtaining the elements of matrix $E$ can be regarded as an especially important goal since, by Eq. (9), they are directly related to matrix elements of $M$ whose determination is our main purpose. From Eq. (19) it can be seen that matrix $R_{A}$ is proportional to matrix $E$. Unfortunately the constant of proportionality will not be obtained directly from the data, and for that reason neither $R_{-1}$ nor $E$ will be eliminated in favor of the other for the time being.

The eigenvalues $\Lambda_{A}$ and $\Lambda_{D}$ are the most directly and accurately obtainable parameters. By Eq. (13) they are simple functions of the eigentunes, and can be found by locating peaks in the Fourier tune spectrum (as well as by another method to be described below). With no coupling, or even if there is coupling but $\mathcal{E}=0$, by Eq. (12), $\operatorname{tr} A$ and $\operatorname{tr} D$ would be equal to $\Lambda_{A}$ and $\Lambda_{D}$ respectively. As above we decline (at least for the time being) to "cheat" in this way. 
Future equations are somewhat simplified by introducing a parameter

$$
\Delta_{\Lambda}=\Lambda_{D}-\Lambda_{A}
$$

and a "small" parameter $\zeta$. When $\zeta$ is negligible, all formulas simplify greatly.

$$
\begin{aligned}
& \operatorname{tr} A=\Lambda_{A}+\zeta \Delta_{\Lambda} \\
& \operatorname{tr} D=\Lambda_{D}-\zeta \Delta_{\Lambda} .
\end{aligned}
$$

In terms of these variables we have

$$
\begin{aligned}
\mathcal{E} & =\zeta(1-\zeta) \Delta_{\Lambda}^{2} \\
\operatorname{det}\left|R_{A}\right| & =\frac{\zeta}{1-\zeta^{\circ}} \\
\mathcal{G} & =\sqrt{\frac{\left|\Lambda_{A}-\operatorname{tr} D\right|}{\left|\Lambda_{A}-\Lambda_{D}\right|}}=\sqrt{1-\zeta} \\
E & =-\Delta_{\Lambda}(1-\zeta) R_{A} \quad \text { or } \\
e=-\Delta_{\Lambda}(1-\zeta) R_{A 11} & ; \quad f=-\Delta_{\Lambda}(1-\zeta) R_{A 12} \text { and } \\
g=-\Delta_{\Lambda}^{\prime}(1-\zeta) R_{A 21} & ; h=-\Delta_{\Lambda}(1-\zeta) R_{A 22}
\end{aligned}
$$

where $\mathcal{G}$, used below, replaces a different symbol that was multiply defined in reference ${ }^{[1]}$.

\section{Transformation To An Eigenbasis}

In order to define Twiss parameters in a coupled lattice it is necessary to perform a linear transformation from the $\mathrm{x}, \mathrm{y}$ basis to an eigenvector basis.

In a two component space basis vectors can be expressed as

$$
\hat{\chi}_{1}=\left(\begin{array}{l}
1 \\
0
\end{array}\right) ; \quad \hat{\chi}_{2}=\left(\begin{array}{l}
0 \\
1
\end{array}\right)
$$

These can be used to define an $\mathrm{x}, \mathrm{y}$ basis in the four component space.

$$
\hat{\imath}^{(1)}=\left(\begin{array}{c}
\hat{\chi}_{1} \\
0
\end{array}\right) ; \quad \hat{\imath}^{(2)}=\left(\begin{array}{c}
\hat{\chi}_{2} \\
0
\end{array}\right) ; \quad \hat{\imath}^{(3)}=\left(\begin{array}{c}
0 \\
\hat{\chi}_{1}
\end{array}\right) ; \quad \hat{\imath}^{(4)}=\left(\begin{array}{c}
0 \\
\hat{\chi}_{2}
\end{array}\right)
$$

Similarly, from (20), a basis of eigenvectors is

$$
\hat{I}^{(1)}=\mathcal{G}\left(\begin{array}{c}
\hat{\chi}_{1} \\
R_{A} \hat{\chi}_{1}
\end{array}\right) ; \hat{I}^{(2)}=\mathcal{G}\left(\begin{array}{c}
\hat{\chi}_{2} \\
R_{A} \hat{\chi}_{2}
\end{array}\right) ; \hat{I}^{(3)}=\mathcal{G}\left(\begin{array}{c}
R_{D} \hat{\chi}_{1} \\
\hat{\chi}_{1}
\end{array}\right) ; \hat{I}^{(4)}=\mathcal{G}\left(\begin{array}{c}
R_{D} \hat{\chi}_{2} \\
\hat{\chi}_{2}
\end{array}\right)
$$

where $\mathcal{G}$ is a numerical factor yet to be determined. These bases are related by 
a linear transformation

$$
\hat{I}^{(k)}=G_{k i} i^{(i)}
$$

where summation is implied. A general vector can be expressed in terms of either basis, yielding the equality

$$
x_{i} i^{(i)}=X_{k} \hat{I}^{(k)}=X_{k} G_{k i} i^{(i)}
$$

and from this the coordinates are related, in component and in matrix notation by

$$
x_{i}=X_{k} G_{k i} ; \quad x=G^{T} X
$$

By substituting from Eq. (27) into Eq. (28) one obtains

$$
G^{T}=\mathcal{G}\left(\begin{array}{cc}
I & R_{D} \\
R_{A} & I
\end{array}\right)
$$

The value of $\mathcal{G}$ given above in Eq. (24) is such as to make $\operatorname{det}\left|G^{T}\right|=1$,

$$
\left(G^{T}\right)^{-1}=\mathcal{G}\left(\begin{array}{cc}
I & -R_{D} \\
-R_{A} & I
\end{array}\right)
$$

and

$$
\bar{G}^{T}=\left(G^{T}\right)^{-1}
$$

which shows that $G$ is symplectic.

In the $x, y$ basis the one turn map relating turns $t$ and $t+1$ is given by

$$
x_{t+1}=M x_{t}
$$

Substituting from Eq. (30) one gets

$$
G^{T} X_{t+1}=M G^{T} X_{t}
$$

which means that the transfer matrix in the transformed basis is

$$
\underline{M}=\left(G^{T}\right)^{-1} M G^{T}=\mathcal{G}^{2}\left(\begin{array}{cc}
I & -R_{D} \\
-R_{A} & I
\end{array}\right)\left(\begin{array}{cc}
A & B \\
C & D
\end{array}\right)\left(\begin{array}{cc}
I & R_{D} \\
R_{A} & I
\end{array}\right) \equiv\left(\begin{array}{cc}
\underline{A} & 0 \\
0 & \underline{D}
\end{array}\right)
$$

from which it follows that

$$
\begin{aligned}
& \underline{A}=\mathcal{G}^{2}\left(A+B R_{A}-R_{D} C-R_{D} D R_{A}\right) \\
& \underline{D}=\mathcal{G}^{2}\left(-R_{A} A R_{D}-R_{A} B+C R_{D}+D\right) .
\end{aligned}
$$

From $\underline{A}$ and $\underline{D}$ the Twiss parameters in the eigenbasis can be extracted. The determinants $\operatorname{det}|\underline{A}|$ and $\operatorname{det}|\underline{D}|$ must both be unity since they are equal to the 
product of eigenvalues, which is one. As a result $A$. for example, can be written in "Twiss form"

$$
\left(\begin{array}{ll}
\underline{A}_{11} & \underline{A}_{12} \\
\underline{A}_{21} & \underline{A}_{22}
\end{array}\right)=\left(\begin{array}{cc}
\cos \mu_{A}+\alpha_{A} \sin \mu_{A} & \beta_{A} \sin \mu_{A} \\
-\gamma_{A} \sin \mu_{A} & \cos \mu_{A}-\alpha_{A} \sin \mu_{A}
\end{array}\right)
$$

where

$$
\mu_{A}=\arccos (\operatorname{tr} \underline{A} / 2)
$$

It is assumed here that any sign ambiguity has already been resolved in Eq. (11). In Eq. (38) the Twiss parameters are obtained from element-by-element comparison; they are

$$
\beta_{A}=\underline{A}_{12} / \sin \mu_{A} ; \quad \gamma_{A}=-\underline{A}_{21} / \sin \mu_{A} ; \quad \alpha_{A}=\left(\underline{A}_{11}-\underline{A}_{22}\right) /\left(2 \sin \mu_{A}\right),
$$

and similarly for $\underline{D}$.

There is a quadratic form which is invariant under the application of the transfer map Eq. (1). Defining $(X, P, Y, Q) \equiv\left(X_{1}, X_{2}, X_{3}, X_{4}\right)$, it and its $D$ counterpart are given by

$$
\begin{aligned}
& \epsilon_{A}=\gamma_{A} X^{2}+2 \alpha_{A} X P+\beta_{A} P^{2} \\
& \epsilon_{D}=\gamma_{D} Y^{2}+2 \alpha_{D} Y Q+\beta_{D} Q^{2}
\end{aligned}
$$

which are the generalizations of the Courant-Snyder invariants. They could be called eigeninvariants or eigenemittances, with the former to be preferred because it tends less to perpetuate the confusion between a single particle parameter and a beam parameter that comes from the use of the symbol $\epsilon$ for both purposes. Because both names are so ugly we will use the term mode invariant instead. A given vector $x$ will, in general, have non-vanishing components in both of the eigenbases. The corresponding mode invariants can be evaluated using the inverse of Eq. (30) to obtain $X$, followed by substitution into Eq. (41).

Finally we wish to characterize each of the eigenbases by a spatial orientation. The points in a Poincaré plot ( $x, y$ projection) are not restricted to a single line; rather they moves along an elliptical trajectory. It is reasonable to characterize the orientation of the $A$-eigenbasis by the angular deviation, $\theta_{A}$, of the major principle axis of the ellipse, away from the $x$-axis, and similarly for $\underline{D}$.

Observing at a fixed point in the lattice where the lattice functions are $\beta_{A}$ and $\alpha_{A}$ the eigenmotion can be described in the "pseudoharmonic" form

$$
\begin{aligned}
X & =\sqrt{\beta_{A} \epsilon_{A}} \cos \psi_{A} \\
P & =\sqrt{\beta_{A} \epsilon_{A}}\left(\sin \psi_{A}-\alpha_{A} \cos \psi_{A}\right) / \beta_{A} .
\end{aligned}
$$

Here $\psi_{A}$ advances by an angle equal to $-\mu_{A}$ on each turn, and eventually, modulo 
$2 \pi$, takes on all values between 0 and $2 \pi$. The factor $\sqrt{\beta_{A} \epsilon_{A}}$, (which was not included in the corresponding equation of reference, $)^{[1]}$ can initially be regarded as an inessential common factor, but it will be of importance both in combining $A$ and $D$ eigenmotions and in comparing signals at different BPM's. Substitution into Eq. (30) permits the motion to be expressed in the form

$$
\begin{aligned}
& x=\mathcal{G} \sqrt{\beta_{A} \epsilon_{A}} \cos \psi_{A} \\
& y=\mathcal{G} e_{A} \sqrt{\beta_{A} \epsilon_{A}} \cos \left(\psi_{A}+\Phi_{A}\right)
\end{aligned}
$$

where

$$
\begin{aligned}
e_{A}^{2} & =\left[R_{A 11}-\left(\alpha_{A} / \beta_{A}\right) R_{A 12}\right]^{2}+\left(R_{A 12} / \dot{\beta_{A}}\right)^{2} \\
\Phi_{A} & =-\arctan \frac{R_{A 12} / \beta_{A}}{R_{A 11}-\left(\alpha_{A} / \beta_{A}\right) R_{A 12}}
\end{aligned}
$$

It can be shown that the angle of orientation of the ellipse is given by

$$
\tan 2 \theta_{A}=-\frac{2\left[R_{A 11}-\left(\alpha_{A} / \beta_{A}\right) R_{A 12}\right]}{1-\left[R_{A 11}-\left(\alpha_{A} / \beta_{A}\right) R_{A 12}\right]^{2}-\left(R_{A 12} / \beta_{A}\right)^{2}}
$$

The orientation of the other mode axis can be found similarly. In general, the two axes are not orthogonal. Normally, since ideal behaviour would have the eigenaxes exactly horizontal and vertical, the deviations of these angles from zero can be regarded as a measure of the seriousness of the coupling.

When two oscillatory quantities are related as $x$ and $y$ are in Eqs. (43), it is natural to relate them by a "transfer function". Since they oscillate at the same frequency, their relationship is completely specified by a ratio of amplitudes, in this case $e_{A}$, and a phase difference, in this case $\Phi_{A}$. The term transfer function is appropriate because the two functions are causally related (otherwise they would not have the same frequency), but one need not "cause" the other. Because the two signals are coherent, the signal-to-noise ratio of their ratio can probably be improved by averaging measurements made at different times. The transfer functions will be obtained by Fourier series analysis in a later section. 
7. Difference Equations Satisfied By the Motion

Using the fact that $M^{-1}$ can be used to propagate backwards in time, relation (8) can be used to obtain four third-order, coupled difference equations that relate the coordinates on three successive turns (labeled $-, 0,+$ ):

$$
\begin{aligned}
& x_{+}-\operatorname{tr} A x_{0}+x_{-}=h y_{0}-f q_{0} \\
& p_{+}-\operatorname{tr} A p_{0}+p_{-}=-g y_{0}+e q_{0} \\
& y_{+}-\operatorname{tr} D y_{0}+y_{-}=e x_{0}+f p_{0} \\
& q_{+}-\operatorname{tr} D q_{0}+q_{-}=g x_{0}+h p_{0} .
\end{aligned}
$$

It is possible to uncouple these equations. Start by squaring Eq. (8);

$$
\left(M+M^{-1}\right)^{2}=M M+2 I+M^{-1} M^{-1}=\left(\begin{array}{cc}
\operatorname{tr}^{2} A+\mathcal{E} & \left(\Lambda_{A}+\Lambda_{D}\right) \bar{E} \\
\left(\Lambda_{A}+\Lambda_{D}\right) \bar{E} & \operatorname{tr}^{2} D+\mathcal{E}
\end{array}\right) \text {. }
$$

From this one obtains fifth order equations linking five successive turns

$$
\begin{aligned}
& x_{++}+\left(2-\operatorname{tr}^{2} A-\mathcal{E}\right) x_{0}+x_{--}=\left(\Lambda_{A}+\Lambda_{D}\right)\left(h y_{0}-f q_{0}\right) \\
& p_{++}+\left(2-\operatorname{tr}^{2} A-\mathcal{E}\right) p_{0}+p_{--}=\left(\Lambda_{A}+\Lambda_{D}\right)\left(-q y_{0}+e q_{0}\right) \\
& y_{++}+\left(2-\operatorname{tr}^{2} D-\mathcal{E}\right) y_{0}+y_{--}=\left(\Lambda_{A}+\Lambda_{D}\right)\left(e x_{0}+f p_{0}\right) \\
& q_{++}+\left(2-\operatorname{tr}^{2} D-\mathcal{E}\right) q_{0}+q_{--}=\left(\Lambda_{A}+\Lambda_{D}\right)\left(g x_{0}+h q_{0}\right) .
\end{aligned}
$$

Substituting from Eq. (46) into Eq. (48) and keeping only the equations for the measured quantities $x$ and $y$ yields

$$
\begin{array}{r}
x_{++}+x_{--}-\left(\Lambda_{A}+\Lambda_{D}\right)\left(x_{+}+x_{-}\right)+\left(2+\Lambda_{A} \Lambda_{D}\right) x_{0}=0 \\
y_{++}+y_{--}-\left(\Lambda_{A}+\Lambda_{D}\right)\left(y_{+}+y_{-}\right)+\left(2+\Lambda_{A} \Lambda_{D}\right) y_{0}=0
\end{array}
$$

What makes these equations truly remarkable in that, in full generality with arbitrary coupling, and with coefficients that are lattice invariants, the same at every BPM, they constrain the output of each plane of every BPM. Since any spurious superimposed signal will not satisfy this constraint, these equations can give a measure of the importance of noise or distortion due to nonlinear electronics in the signal processing or distortion due to nonlinear motion. Eqs. (49) generalize the following difference equations for uncoupled motion

$$
\begin{aligned}
& x_{+}-\Lambda_{x} x_{0}+x_{-}=0 \\
& y_{+}-\Lambda_{y} x_{0}+y_{-}=0
\end{aligned}
$$

It can be shown that with no coupling Eqs. (49) and (50) are consistent. The first of Eqs. (50) can be regarded as a "trigonometric" identity relating, in $2 \mathrm{D}\left(x, x^{\prime}\right)$ 
phase space, projections of a radius vector that advances through a constant angle $\mu_{x}$ each turn. Eqs. (49) relate similar projections in 4D phase space.

If there were no noise or distortion, and if the $x$ and $y$ measurements were arbitrarily accurate, Eqs. (49) could be used to determine both eigentunes to arbitrarily high accuracy from as few as five turns worth of data.

The eigentunes can be obtained by applying Eq. (49) to actual data. Define the expectation value $\langle f\rangle$ of $N$ samples $f_{i}$ by $\sum_{i}^{n} f_{i} / N$. Multiplying the two equations of (49) by $x_{0}$ and $y_{0}$ respectively, taking expectation values, and rearranging to express as equations for $\Lambda_{A}$ and $\Lambda_{D}$ yields

$$
\begin{aligned}
\left(\begin{array}{cc}
<\left(x_{+}+x_{-}\right) x_{0}> & -<x_{0}^{2}> \\
<\left(y_{+}+y_{-}\right) y_{0}>-<y_{0}^{2}>
\end{array}\right) & \left(\begin{array}{c}
\Lambda_{A}+\Lambda_{D} \\
\Lambda_{A} \Lambda_{D}
\end{array}\right) \\
& =\left(\begin{array}{c}
<\left(x_{++}+x_{--}\right) x_{0}>+2<x_{0}^{2}> \\
<\left(y_{++}+y_{--}\right) y_{0}>+2<y_{0}^{2}>
\end{array}\right) .
\end{aligned}
$$

When this equation is used to extract eigentunes for the simulated data, the values agree well with peak locations obtained from Fourier analysis: $\nu_{A}$ (diff.eq.) $=$ $\nu_{A}$ (Fourier) $\pm 0.00005 ; \nu_{D}$ (diff.eq.) $=\nu_{D}$ (Fourier) \pm 0.003 . The equation is less accurate when it is applied to measured data : $\nu_{A}$ (diff.eq.) $=\nu_{A}$ (Fourier) \pm $0.001 ; \nu_{D}$ (diff.eq.) $=\nu_{D}$ (Fourier) \pm 0.004 . It is not suprising that the accuracy for $\nu_{D}$ is not as good as for $\nu_{A}$, since vertical oscillations should by design not be present at all. The fact that the accuracy is better for simulated data than for actual data is due to noise or signal processing distortion. While Fourier analysis yields optimal filtering, the difference equation estimates are likely to be biased by noise signals. Our analysis procedure is to use the difference equation to obtain tentative tune values. From this an improved value is obtained by obtaining Fourier amplitudes at points on a fine nearby grid and interpolating to find the maximum. Tunes determined in that way are shown in Fig. 2. The way error bars are determined will be described below. In most cases in these plots they are too small to be visible. These tune values were used in evaluating the Fourier coefficients defined in the next section. Most relationships will be expressed in terms of Fourier coefficients since that tends to incorporate the benefits of filtering in the frequency domain. 

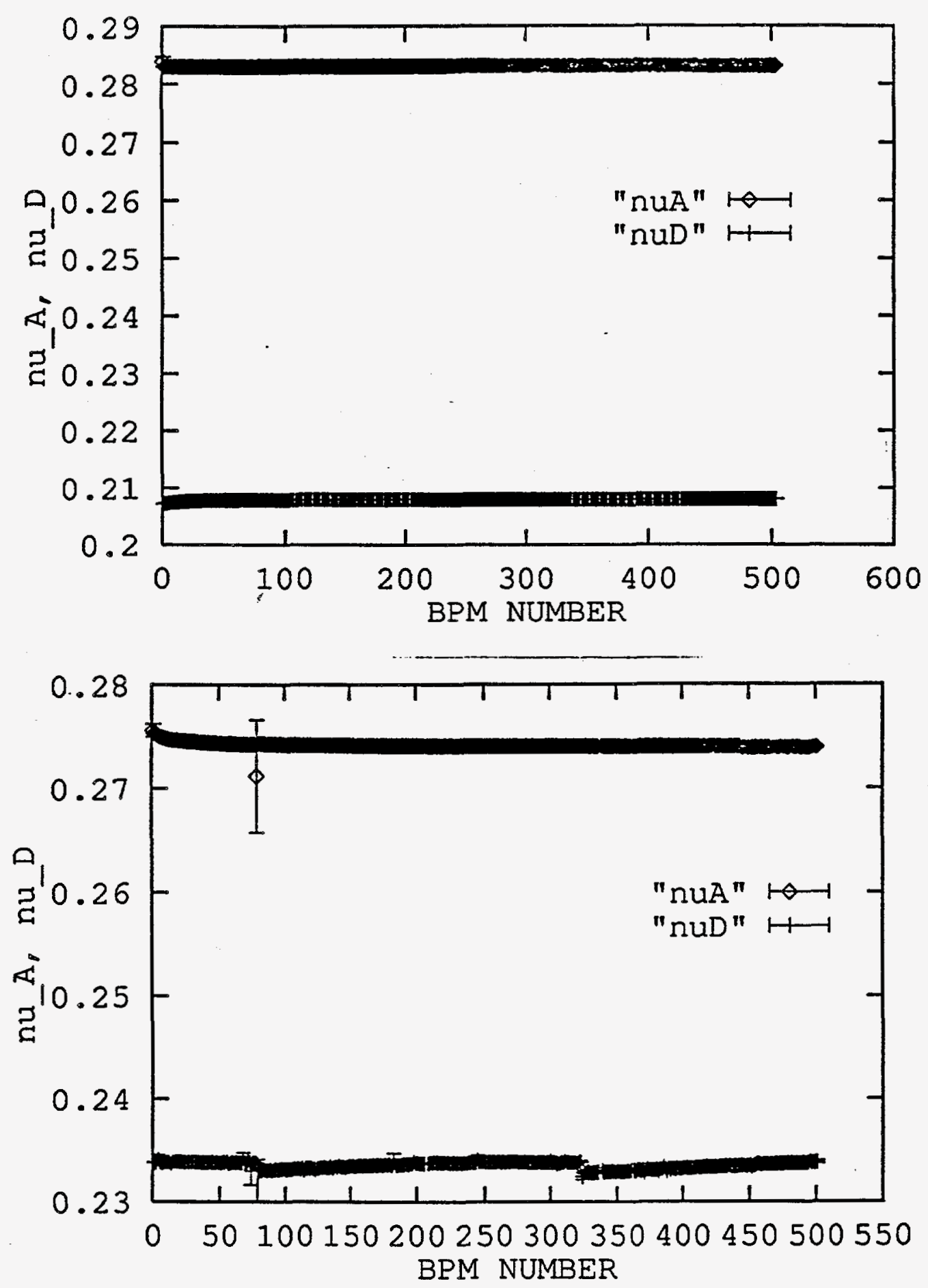

Figurc 2. Mode tunes $\nu_{A}$ and $\nu_{D}$, as determined at all 504 BPM's. Upper figure is from simulation, lower figure from measured data. 


\section{Extraction of Fourier Coefficients}

In complete generality linear $x$ and $y$ motions can be expressed as superpositions of $A$-type and $D$-type motions. The simplest possible description is in "normalized eigencoordinates" $\tilde{X}=(\tilde{X}, \tilde{P}, \tilde{Y}, \tilde{Q})^{T}$ for which the phase space motion is circular

$$
\begin{array}{ll}
\tilde{X}=\epsilon_{A}^{1 / 2} \cos \mu_{A} t ; & \tilde{P}=\epsilon_{A}^{1 / 2} \sin \mu_{A} t \\
\tilde{Y}=\epsilon_{D}^{1 / 2} \cos \mu_{D} t ; & \tilde{Q}=\epsilon_{D}^{1 / 2} \sin \mu_{D} t .
\end{array}
$$

Here, and wherever it appears in this report, the symbol $t$ can be regarded either as time, measured in units of the revolution period, or as a turn index that increments by 1 each turn. In laboratory $x, y$ coordinates the motion manifests itself in the form

$$
\begin{aligned}
& x_{t}=A_{x} \cos \mu_{A} t+D_{x c} \cos \mu_{D} t+D_{x s} \sin \mu_{D} t \\
& y_{t}=D_{y} \cos \mu_{D} t+A_{y c} \cos \mu_{A} t+A_{y s} \sin \mu_{A} t .
\end{aligned}
$$

Here the subscript $x$ or $y$ distinguishes between $x$ or $y$ motion, subscript $c$ or $s$ goes with the cos or sinfunction, and the capitalized symbols distinguish between $A$ and $D$ eigenmotions. It is assumed that $\mu_{A}$ and $\mu_{D}$ are incommensurate (i.e. not rationally related; i.e. non-resonant) which means there is a time at which the nominally dominant coordinate for the two eigenmotions are simultaneously maximum. Such a time origin has been chosen in (53) (different in general from that in (52)) so that the sin term is missing for $x$ in the $A$ eigenmotion and for $y$ in the $D$ eigenmotion. For that reason the subscript $c$ has been left off $A_{x}$ and $D_{y}$. Also we can assume $A_{x}$ and $D_{y}$ are positive without loss of generality.

All six of the coefficients in Eq. (53) are easily and accurately extracted from the turn-by-turn data by Fourier analysis.

For brevity we also define coefficients

$$
A_{y}=\sqrt{A_{y c}^{2}+A_{y s}^{2}} ; \quad D_{x}=\sqrt{D_{x c}^{2}+D_{x s}^{2}} .
$$

In terms of these coefficients, the transfer function ratios and phases of Eqs. (44) are

$$
\begin{array}{rlrl}
e_{A} & =\frac{A_{y}}{A_{x}} ; & \Phi_{A}=-\arctan \frac{A_{y s}}{A_{y c}} \\
e_{D} & =\frac{D_{x}}{D_{y}} ; & \Phi_{D} & =-\arctan \frac{D_{x s}}{D_{x c}} .
\end{array}
$$

It has been implicitly assumed that there is no damping. In fact, for the actual LEP data there is damping with damping decrement $\delta$ (fractional loss per 
turn) of a few parts per thousand. Furthermore it is found that the damping is different for the two modes. Because of damping, the coefficients $A$ and $D$, which would otherwise be constant exhibit time dependence

$$
\begin{aligned}
& A(t)=A(0) e^{-\delta_{A} t} \\
& D(t)=D(0) e^{-\delta_{D} t} .
\end{aligned}
$$

For the LEP data set $14-18$, with $t=1$ revolution,

$$
\begin{aligned}
& e^{-\delta_{A}}=0.9982 \pm 0.0002 \\
& e^{-\delta_{D}}=0.9970 \pm 0.0003
\end{aligned}
$$

The fits of the form (56) are not perfect, and the error assignments in (57) are only rough, but since the damping is so weak it seems legitimate to simply divide the Fourier coefficients by the exponential factors in (56) and then proceed as if there were no damping. That is what has been done and we have chosen to do it implicitly rather than cluttering the equations of this report with explicit $e^{-\delta t}$ factors.

Because the damping in the $D$ mode is appreciably greater than in the $A$ mode (the reason is not known, but the result is consistent with "head-tail damping" that would be expected to be greater in the vertical than in the horizontal plane), and because the $D$ amplitudes are initially smaller than the $A$ amplitudes, the $D / A$ ratio has become really very small after 1000 turns. For this reason the entire analysis reported here is restricted to the first 600 turns. In order to estimate errors for the various parameters these 600 turns were broken into 4 sets of 150 turns each. Identical and completely independent analyses were applied to each 150-turn set; values and errors quoted or plotted are the means and r.m.s. values of the four sets. These errors indicate the variation of these four determinations; they do not indicate the measurement error of the best parameter determination that could be extracted from the data sample.

The transfer function parameters listed in Eqs. (55), evaluated from the coefficients defined in Eqs. (53), with errors calculated as just described, are plotted in Figs. 3-6. Though these functions are rather jerky and discontinuous, it will be seen below that appropriate combinations of them are much more regular. 

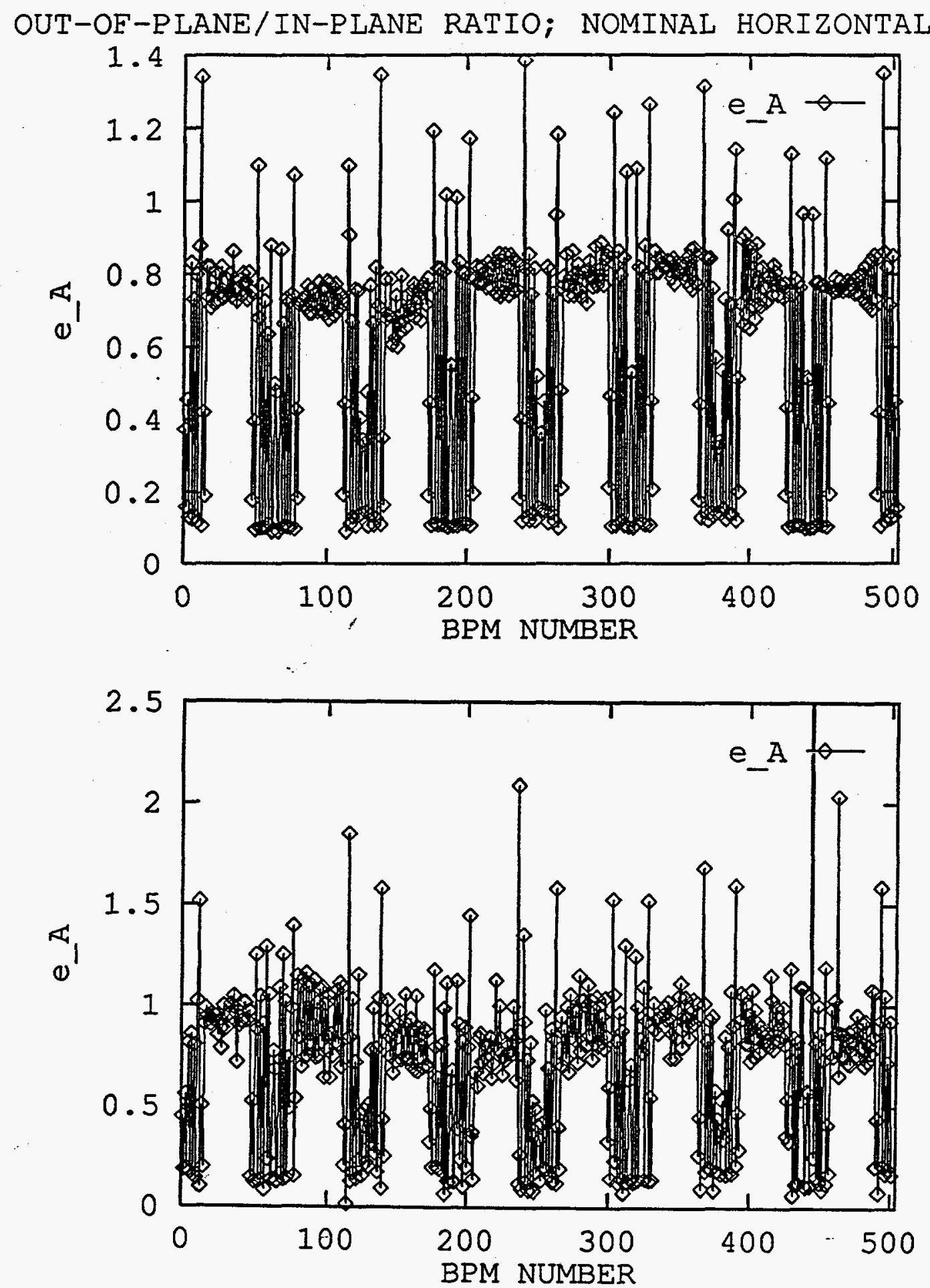

Figure 3. A-mode transfer function amplitude ratio $e_{A}$ evaluated at every BPM. Upper figure is from simulation, lower figure from measured data. 
PHASE OF VERTICAL IN NOMINALLY HORIZONTAL EIGENMOTION
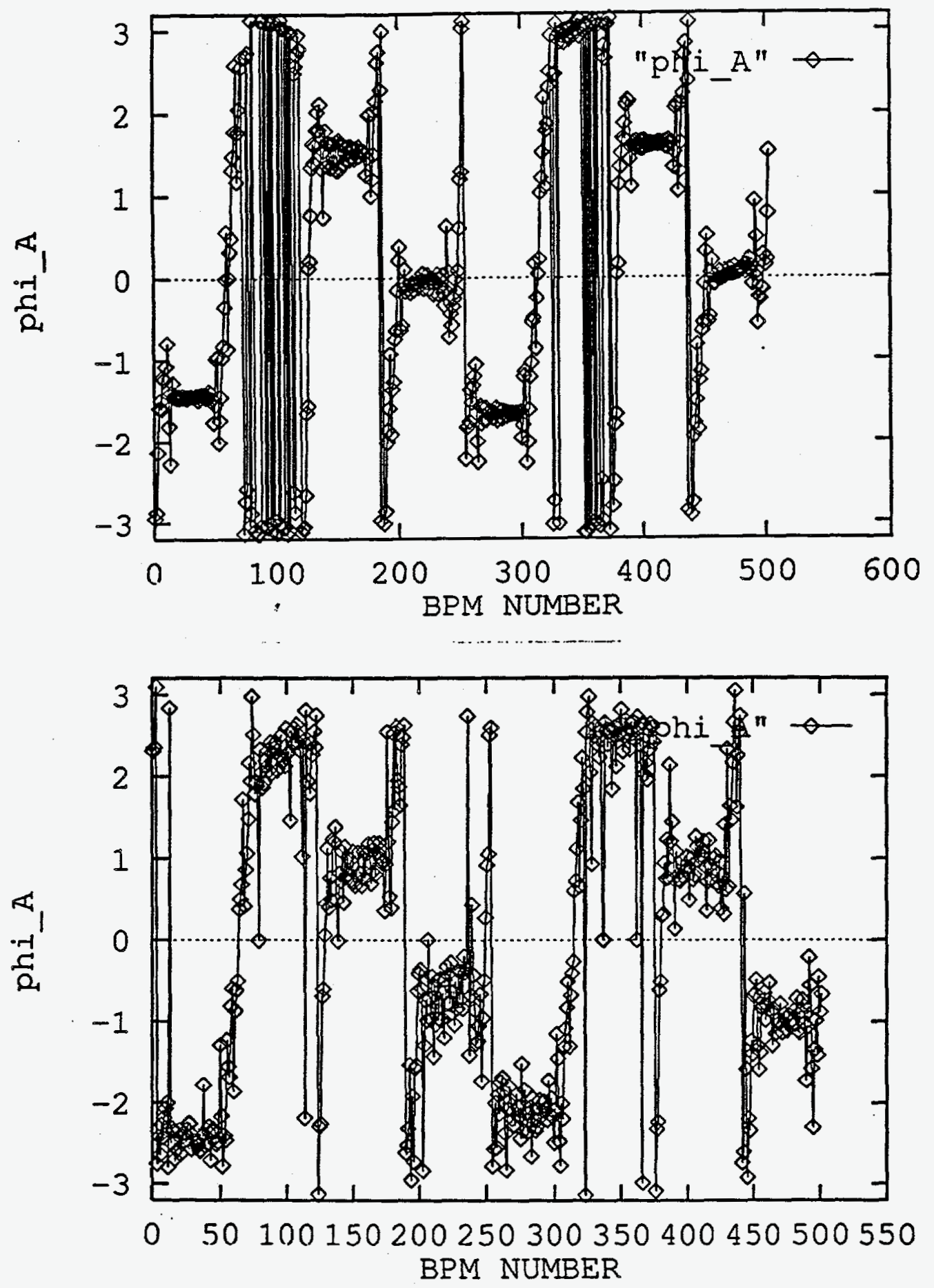

Figure 4. A-mode transfer function phase shift $\Phi_{A}$ evaluated at every BPM. Upper figure is from simulation, lower figure from measured data. 

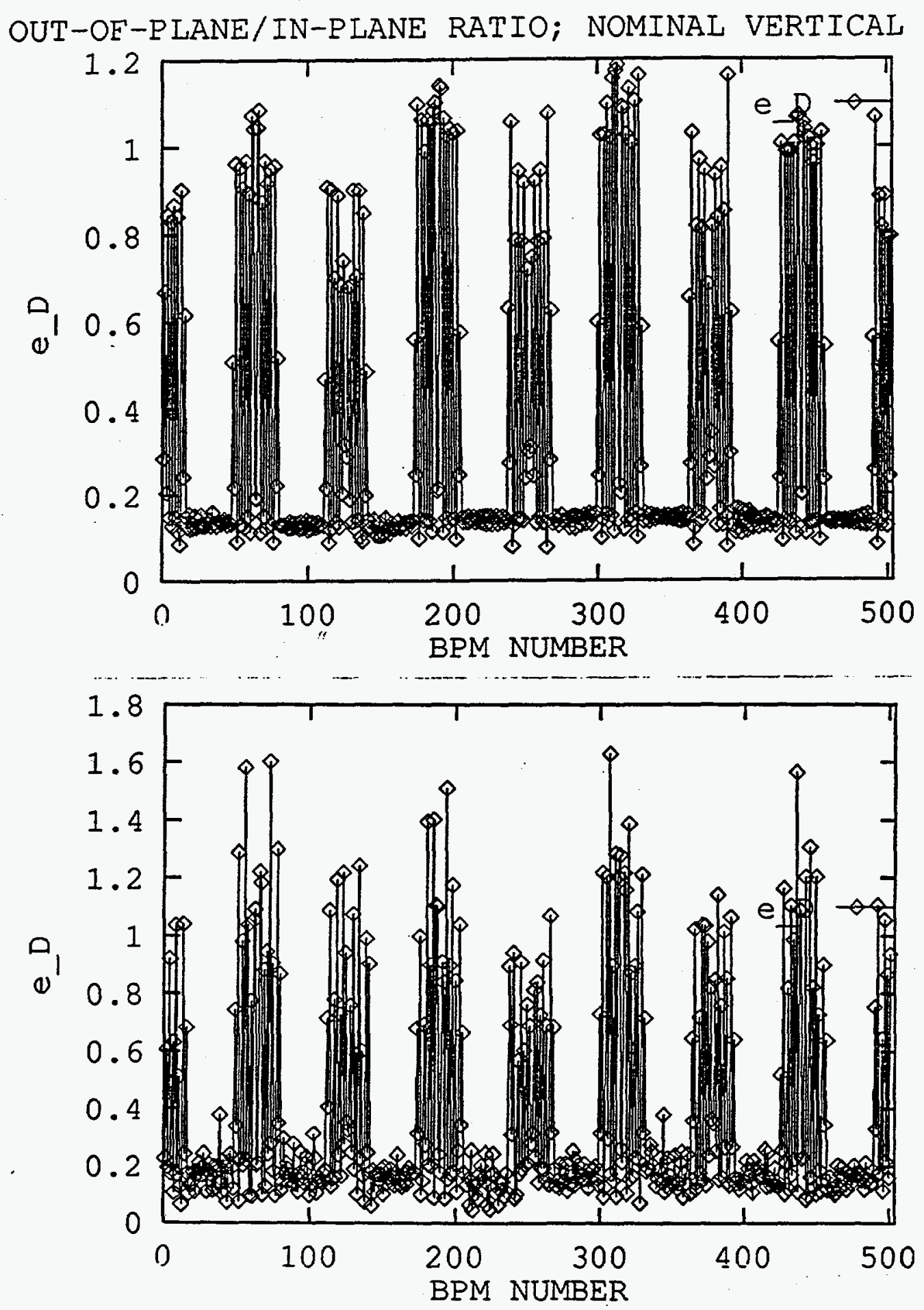

Figure 5. D-mode transfer function amplitude ratio $e_{D}$ evaluated at every BPM. Upper figure is from simulation, lower figure from measured data. 

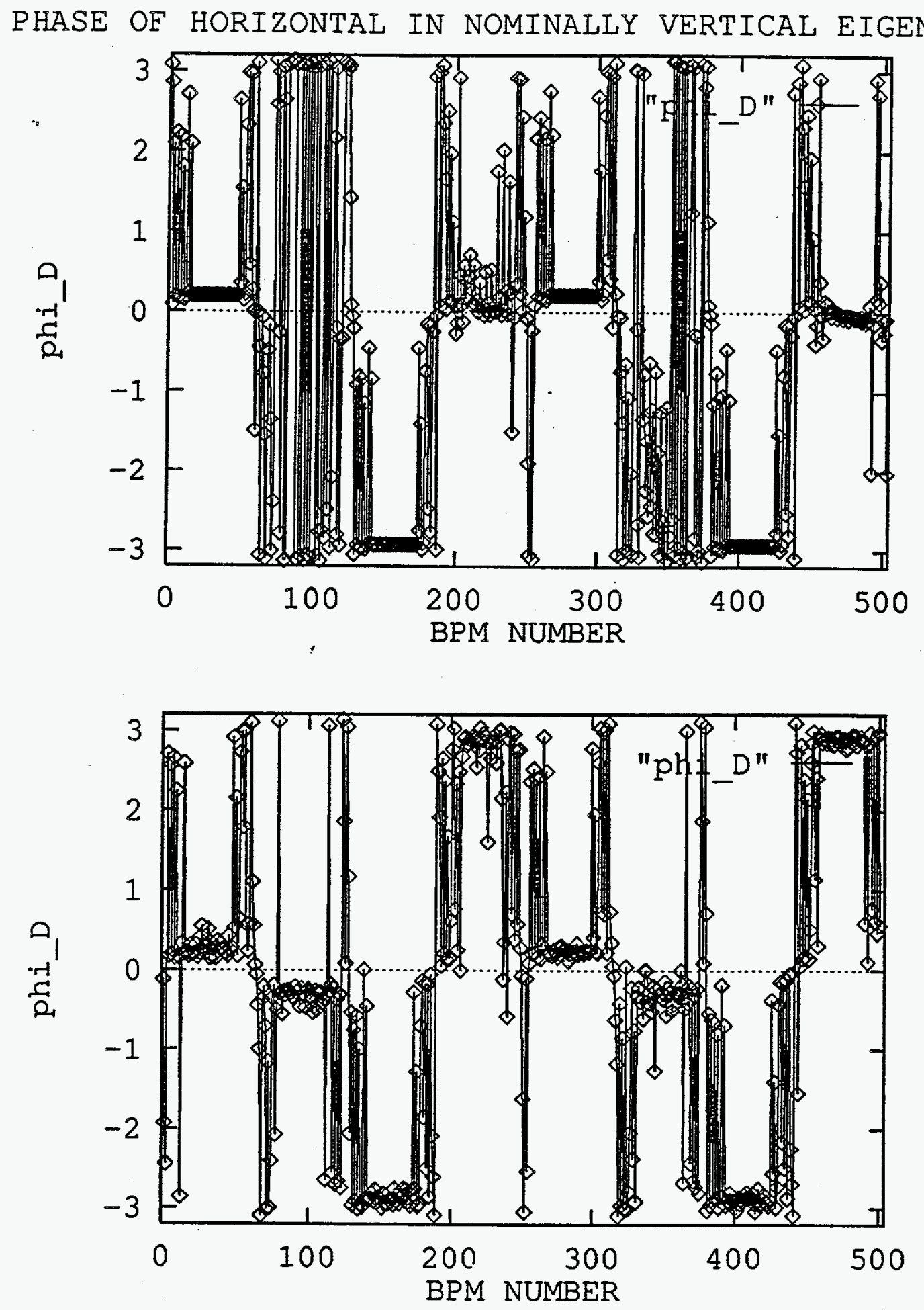

Figure 6. D-mode transfer function phase shift $\Phi_{D}$ evaluated at every BPM. Upper figure is from simulation, lower figure from measured data. 


\section{Relations Between Fourier Coefficients and Matrix Elements}

(Some formulas in this section have already been given at the end of Section 6 . They are repeated here because the simultaneous analysis of both $A$ and $D$ modes is essential.)

The normalized eigencoordinates $\tilde{X}$ introduced in the previous section are related to the eigencoordinates $X=(X, P, Y, Q)^{T}$ of Eq. (30) by a transformation

$$
X=\left(\begin{array}{cc}
\mathcal{B}_{A}^{-1} & 0 \\
0 & \mathcal{B}_{D}^{-1}
\end{array}\right) \tilde{X}
$$

where

$$
\mathcal{B}_{A}=\left(\begin{array}{cc}
\beta_{A}^{-1 / 2} & 0 \\
\alpha_{A} \beta_{A}^{-1 / 2} & \beta_{A}^{1 / 2}
\end{array}\right) ; \quad \mathcal{B}_{A}^{-1}=\left(\begin{array}{cc}
\beta_{A}^{1 / 2} & 0 \\
-\alpha_{A} \beta_{A}^{-1 / 2} & \beta_{A}^{-1 / 2}
\end{array}\right)
$$

and similarly for $D$. Combining these with Eq. (31) yields

$$
x=\mathcal{G}\left(\begin{array}{cc}
I & -\bar{R}_{A} \\
R_{A} & I
\end{array}\right)\left(\begin{array}{cc}
\mathcal{B}_{A}^{-1} & 0 \\
0 & \mathcal{B}_{D}^{-1}
\end{array}\right) \tilde{X} .
$$

Substituting from (52), (58), and (59) into (60) yields

$$
\begin{aligned}
x_{t} & =\mathcal{G} \sqrt{\beta_{A} \epsilon_{A}} \cos \mu_{A} t \\
& +\mathcal{G}\left(\beta_{D}^{1 / 2} R_{A 22}-\alpha_{D} \beta_{D}^{-1 / 2} R_{A 12}\right) \epsilon_{D}^{1 / 2} \cos \mu_{D} t+\mathcal{G} \beta_{D}^{-1 / 2} R_{A 12} \epsilon_{D}^{1 / 2} \sin \mu_{D} t \\
y_{t} & =\mathcal{G} \sqrt{\beta_{D} \epsilon_{D}} \cos \mu_{D} t \\
& +\mathcal{G}\left(\beta_{A}^{1 / 2} R_{A 11}-\alpha_{A} \beta_{A}^{-1 / 2} R_{A 12}\right) \epsilon_{A}^{1 / 2} \cos \mu_{A} t+\mathcal{G} \beta_{A}^{-1 / 2} R_{A 12} \epsilon_{A}^{1 / 2} \sin \mu_{A} t
\end{aligned}
$$

We are now in a position to evaluate selected matrix elements from the measured Fourier coefficients. By identifying coefficients of Eqs. (61) with those of Eq. (53) we obtain $A$-relations

$$
\begin{aligned}
& \frac{A_{y c}}{A_{x}}=R_{A 11}-\frac{\alpha_{A}}{\beta_{A}} R_{A 12} ; \quad \frac{A_{y s}}{A_{x}}=\frac{1}{\beta_{A}} R_{A 12} \\
& R_{A 12}=\beta_{A} \frac{A_{y s}}{A_{x}} ; \quad R_{A 11} \simeq \frac{A_{y c}}{A_{x}}+\alpha_{x} \frac{A_{y s}}{A_{x}}
\end{aligned}
$$

and $D$-relations

$$
\begin{aligned}
\frac{D_{x c}}{D_{y}}=-R_{A 22}-\frac{\alpha_{D}}{\beta_{D}} R_{A 12}^{\prime} ; & \frac{D_{x s}}{D_{y}}=\frac{1}{\beta_{D}} R_{A 12}^{\prime} \\
R_{A 12}^{\prime}=\beta_{D} \frac{D_{x s}}{D_{y}} ; & R_{A 22} \simeq-\frac{D_{x c}}{D_{y}}-\alpha_{y} \frac{D_{x s}}{D_{y}}
\end{aligned}
$$

where the equations for $R_{A 11}$ and $R_{A 22}$ are indicated only as approximations, 
reflecting the fact that the replacements $\alpha_{-t} \rightarrow \alpha_{x}$ and $\alpha_{D} \rightarrow \alpha_{y}$, valid only perturbatively, have had to be made since we will not succeed in extracting $\alpha_{A}$ and $\alpha_{D}$ from the data. (One could hope for the terms containing $\alpha_{A}$ and $\alpha_{D}$ to be small, but unfortunately in general the two terms of $R_{A 11}$ and $R_{A 22}$ have comparable numerical magnitudes.) A prime has been added to $R_{A 12}^{\prime}$ in the $D$-equations to indicate that, although in the formalism it is the same as $R_{A 12}$, its determination from data using Eqs. (63) is independent of its determination using Eqs. (62). Until $\beta_{A}$ and $\beta_{D}$ have been extracted this redundancy check is not quite ideal since they have to be approximated by the ideal values $\beta_{x}$ and $\beta_{y}$. In a later section $\beta_{A}$ and $\beta_{D}$ will be obtained directly from the data in an almost model-independent way and we will use those values for the present analysis. It is possibile for both $R_{A 12}^{\prime}$ and $R_{A 12}$ to vanish or be small, and at such points the ratio is indeterminate or poorly determined. For that reason, in Fig. 7, paired values of $R_{A 12}$ as abscissa and $R_{A 12}^{\prime}$ as ordinate, are plotted. In this plot the redundancy check reduces to the requirement that all points lie on a straight line. Simulated data is in the upper figure, measured data in the lower. There is near-perfect agreement for the simulated data, nicely satisfying the redundancy check. The ratios also cluster around the best-fit straight line for the actual data; deviations could perhaps be used for estimating the errors in the matrix element determinations.

The equations of this section partially substantiate the statement made previously that three of the four elements of $R_{A}$ are directly measurable, but that $R_{A 21}$ is not. The determination cannot be completed in a model-independent way until the perturbed Twiss functions are determined. Unfortunately, since slope variables $p$ and $q$ are not measured, we will not succeed in extracting $\alpha_{A}$ and $\alpha_{D}$. Also the presence of noise could cause the derived values of $\beta_{A}$ and $\beta_{D}$ to be even less valid than $\beta_{x}$ and $\beta_{y}$ but, "planning for success", we hope this is not the case. 

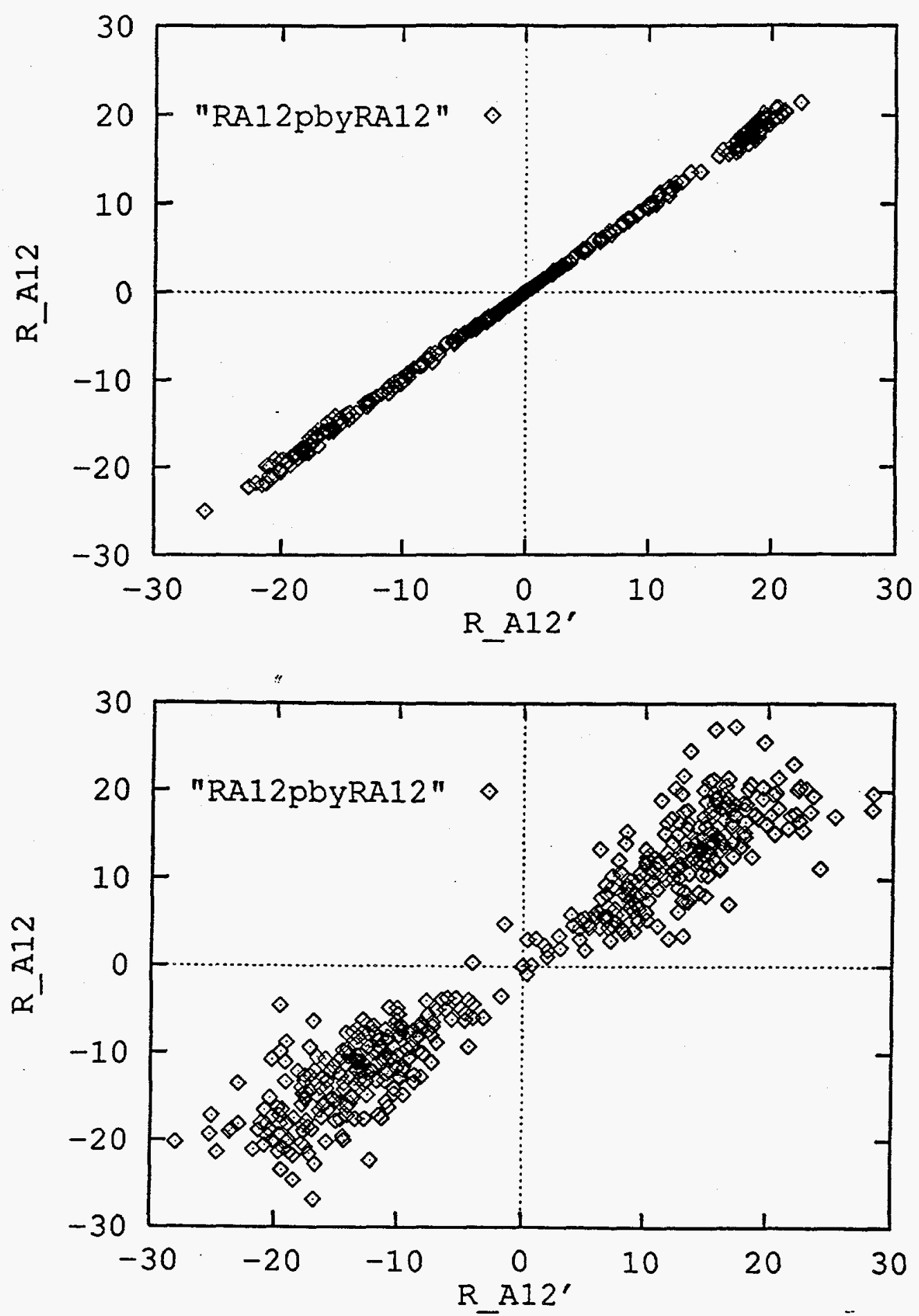

Figure 7. A point is plotted at every BPM with $R_{A 12}$ as abscissa and $R_{A 12}^{\prime}$ as ordinate. Since these are two independent determinations of the same matrix element, all points should lie on the main diagonal. Upper figure is from simulation, lower figure from measured data. 
10. Determination of Mode Invariants and the "Magic Ratio"

As in Eq. (43), the measured displacements $(x, y)$ can be be expressed in terms of the mode invariants defined in Eqs. (41) and the measured transfer function parameters of Eqs. (55).

$$
\begin{aligned}
x_{t} & =\mathcal{G} \sqrt{\beta_{A} \epsilon_{A}} \cos \mu_{A} t+\mathcal{G} e_{D} \sqrt{\beta_{D} \epsilon_{D}} \cos \left(\mu_{D} t+\Phi_{D_{x}}\right) \\
y_{t} & =\mathcal{G} e_{A} \sqrt{\beta_{A} \epsilon_{A}} \cos \left(\mu_{A} t+\Phi_{A y}\right)+\mathcal{G} \sqrt{\beta_{D} \epsilon_{D}} \cos \mu_{D} t
\end{aligned}
$$

where the two newly-introduced phases will not be important. These equations are the same as Eqs. (53) except for the symbols used for coefficients. We equate the coefficients, explicitly indicating now that the Fourier coefficients and the $\beta$-functions depend on longitudinal coordinate $s$ while the mode invariants $\epsilon_{A}$ and $\epsilon D$ do not;

$$
\sqrt{\epsilon}_{A}=\frac{A_{x}(s)}{\mathcal{G} \sqrt{\beta_{A}(s)}} ; \quad \sqrt{\epsilon_{D}}=\frac{D_{y}(s)}{\mathcal{G} \sqrt{\beta_{D}(s)}},
$$

or expressing these as formulas for the unknown $\beta$-functions,

$$
\beta_{A}(s)=\frac{A_{x}^{2}(s)}{\mathcal{G}^{2} \epsilon_{A}} ; \quad \beta_{D}(s)=\frac{D_{y}^{2}(s)}{\mathcal{G}^{2} \epsilon_{D}} .
$$

We are now in position to derive the advertised "magic ratio". Consistency of Eqs. (62) and (63) requires $R_{A 12}=R_{A 12}^{\prime}$ or

$$
\beta_{A} \frac{A_{y s}}{A_{x}}=\beta_{D} \frac{D_{x s}}{D_{y}}
$$

which can be used to determine the ratio $\beta_{A} / \beta_{D}$. Setting this equal to the ratio of Eqs. (66) yields the invariant

$$
\mathcal{R}_{m} \equiv \frac{\epsilon_{D}}{\epsilon_{A}}=\frac{D_{x s} D_{y}}{A_{y s} A_{x}}
$$

where the ratio $\mathcal{R}_{m}$, none other than the ratio of mode invariants, is "magic" because it is the same for all BPM's and is directly obtained from readily and accurately measurable Fourier coefficients.

There is a tiniest of potential blemishes on this attractive form. The denominator factor $A_{y s} A_{x}$ is capable of vanishing, which can make the ratio at best indeterminate and at worst divergent. Analytically the ratio is indeterminate but for actual data the presence of noise or other distortion makes it possible 
for the ratio to become very large. The ratio has no practical use at positions where this indeterminacy is serious. For this reason, in Fig. 8, we plot numerator factor $D_{x s} D_{y}$ versus denominator factor $A_{y s} A_{x}$, and make a linear regression fit to the resultant dot plot. The simulated data is almost perfectly consistent with the constancy of $\mathcal{R}_{m}$. The actual 14-18 data is also nicely consistent with the linear fit. Except in the indeterminate region near the origin, deviations from constancy of $\mathcal{R}_{m}$ are mostly less than $20 \%$. The superiority of this fit compared to the $R_{A 12}^{\prime} / R_{A 12}$ fit of Fig. 7 suggests that the assumption of unperturbed $\beta$ functions was unjustified there. 
LINEAR REGRESSION DETERMINATION OF "MAGIC RATIO"
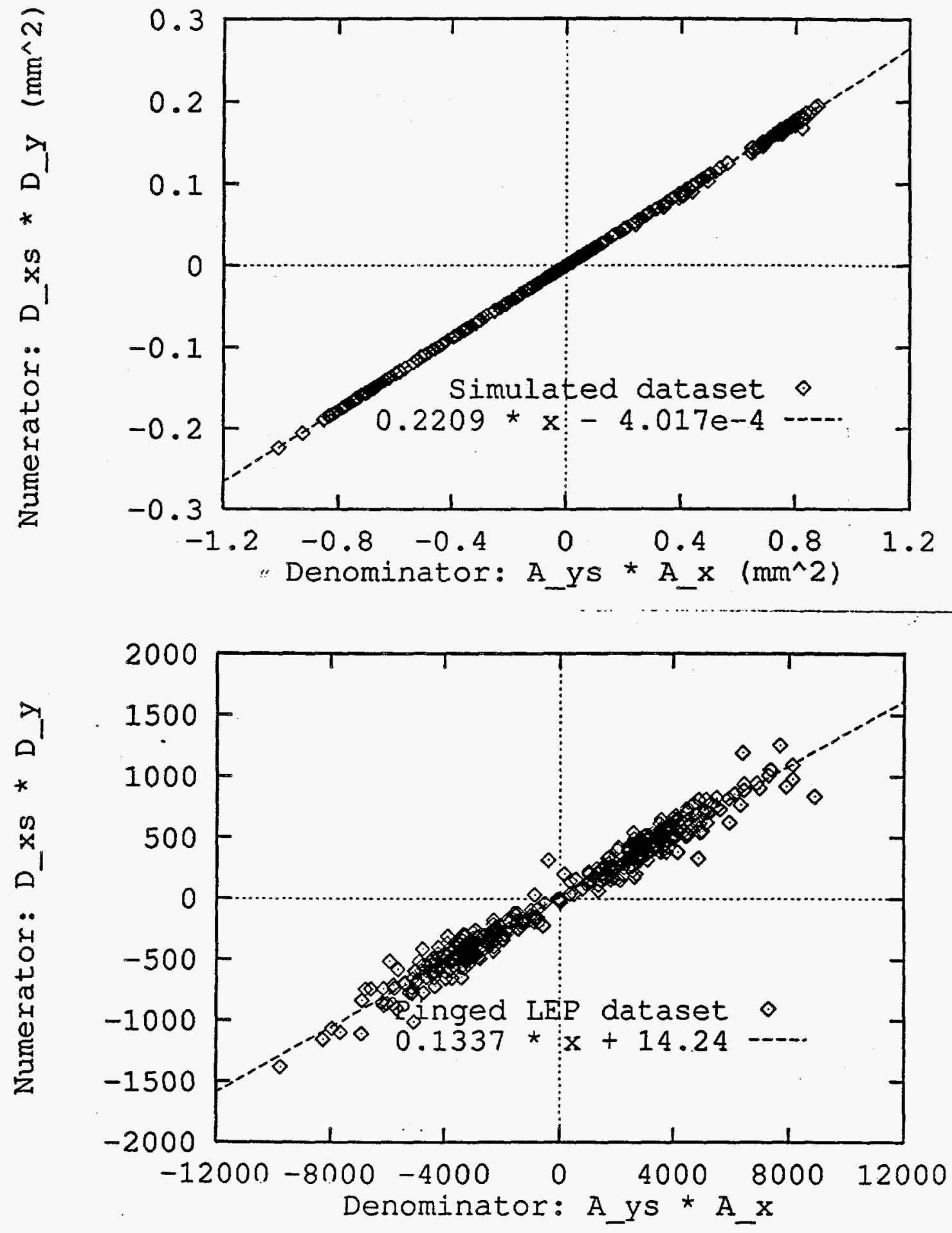

Figure 8. Magic ratio determination. Upper figure is from simulation, lower figure from measured data. 
Once $\mathcal{R}_{m}$ has been made available as the slope of a linear fit in Fig. 8 , it is possible to write the formulas of Eq. (66) in an improved form with only a single unknown common factor $\left(\mathcal{G}^{2} \epsilon_{A}\right)^{-1}$;

$$
\beta_{A}(s)=\frac{1}{\mathcal{G}^{2} \epsilon_{A}} A_{x}^{2}(s) ; \quad \beta_{D}(s)=\frac{1}{\mathcal{R}_{m}} \frac{1}{\mathcal{G}^{2} \epsilon_{A}} D_{y}^{2} .
$$

To this point lattice functions $\beta_{A}$ and $\beta_{D}$ have been unknown and, in a pure sense, they still are because the factor $\left(\mathcal{G}^{2} \epsilon_{A}\right)^{-1}$ is not yet known. But it is so valuable to know the perturbed $\beta$-functions that we now reduce our standards slightly and assume $\left(A_{x}\right.$ being better determined than $D_{y}$ )

$$
\mathcal{G} \epsilon_{A}^{1 / 2}=<\frac{A_{x}(s)}{\beta_{x}^{1 / 2}(s)}>
$$

Taken with Eq. (69), this is similar to assuming

$$
<\beta_{A}^{-1}>=<\beta_{x}^{-1}>\text {; }
$$

i.e. the average inverse $\beta$-function is unperturbed, which is based on preserving the equality of $\int d s / \beta_{x}(s)$ and the full (integer part plus fractional part) tune of the lattice; that tune is almost surely unperturbed to any relevant accuracy. But relation (71) is compromised by the fact that the BPM distribution around the ring is not uniform. This is especially true for the actual LEP data being analysed, since LEP's BPM's are intentionally placed at points in the lattice where $\beta_{y}$ is maximum. Also $\left\langle\beta_{A}^{-1}\right\rangle$ can be strongly biased by a single bad BPM that yields an anomalously small value of $\beta_{A}$ because $A_{x}$ is small. Anyway Eq. (70) is at least as good as any perturbative relation, and we adopt it tentatively. In any case an error here causes only an overall distance scale error.

Plotted in Fig. 9 are $\beta$-functions determined this way. In this case the simulated data (top graph) and real data (bottom graph) are processed somewhat differently. For the simulated data, since the true perturbed functions are known we plot $\sqrt{\beta_{A, D} / \beta_{A, D} \text { (true) }}$. For the measured data the perturbed $\beta$-function is unknown and we plot $\sqrt{\beta_{A, D} / \beta_{x, y}(s)}$. The deviations from 1 are much greater for the actual data than for the simulated data. It is not known whether that is due to noise or to true deviations of the $\beta$-functions. 
SQ.RT. (BETA FUNCTIONS/TRUE BETA FUNCTIONS)

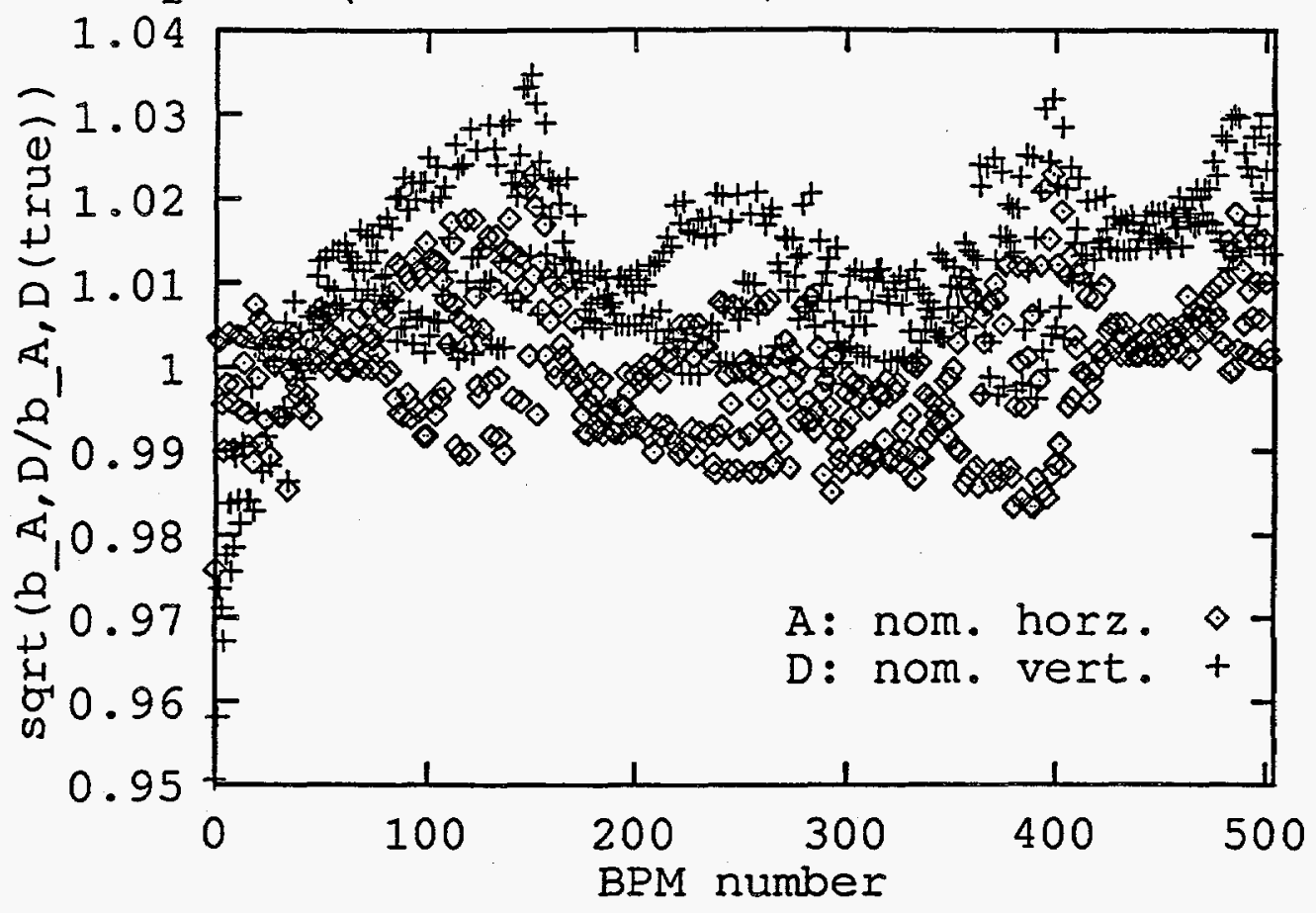

SQ.RT. (BETA FUNCTIONS/IDEAL BETA FUNCTIONS)

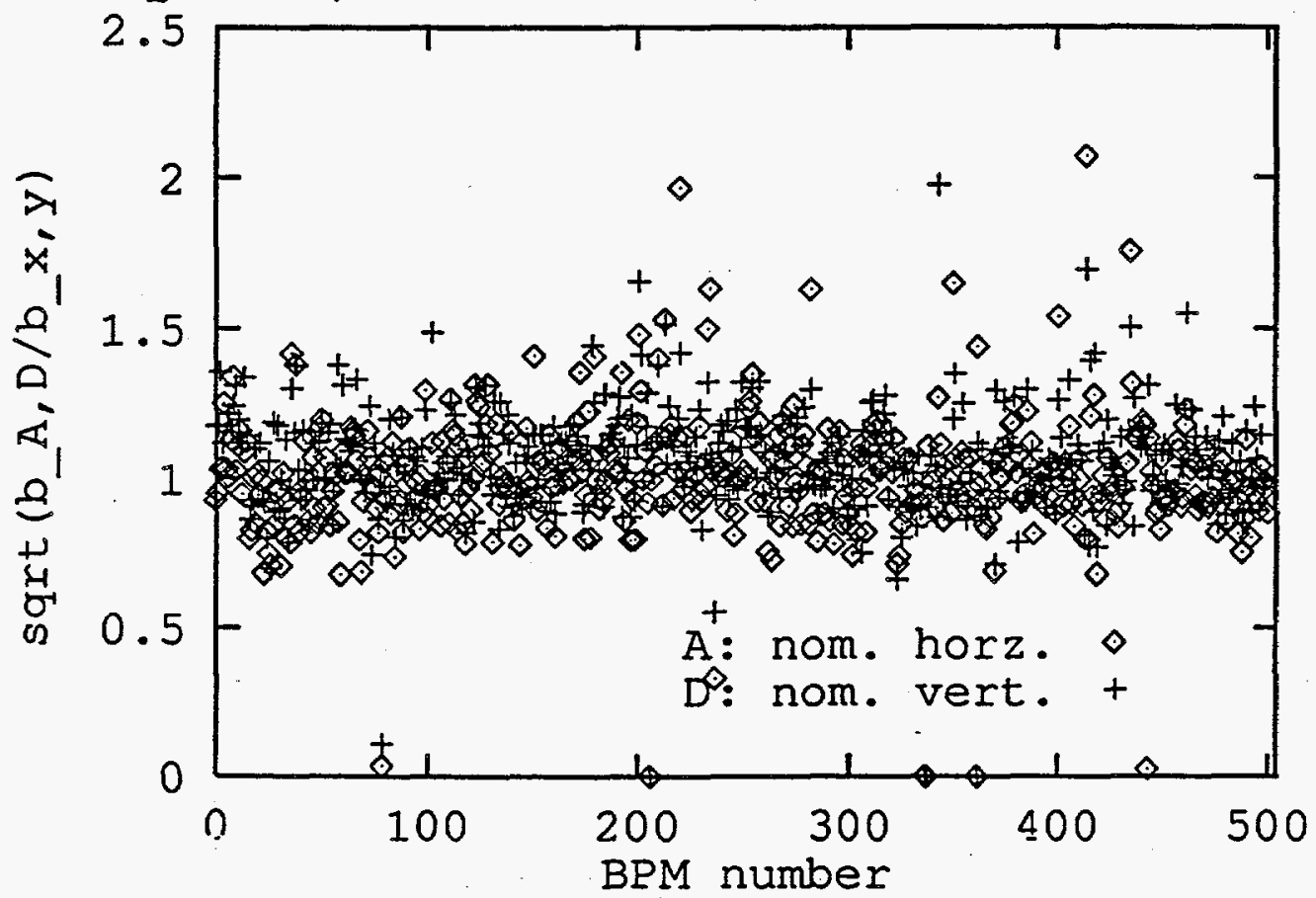

Figure 9. (Measured/ideal) $\beta$-function ratios evaluated at every BPM. Upper figure is $\sqrt{\beta_{A, D} / \beta_{A, D} \text { (true) }}$ from simulation, lower figure is $\sqrt{\beta_{A, D} / \beta_{x, y}(s)}$ from measured data. 


\section{Attempted Further Parameter Determination}

We substitute all presently known parameters into Eqs. (60) and (53) to obtain

$$
\begin{aligned}
\left(\begin{array}{l}
x_{t} \\
p_{t} \\
y_{t} \\
q_{t}
\end{array}\right)= & \left(\begin{array}{cccc}
1 & 0 & \frac{D_{x c}}{D_{y}}+\alpha_{D} \frac{D_{x s}}{D_{y}} & \beta_{A} \frac{A_{y s}}{A_{x}} \\
0 & 1 & R_{A 21} & -\frac{A_{y c}}{A_{x}}-\alpha_{A} \frac{A_{y s}}{A_{x}} \\
\frac{A_{y c}}{A_{x}}+\alpha_{A} \frac{A_{y s}}{A_{x}} & \beta_{A} \frac{A_{y s}}{A_{x}} & 1 & 0 \\
R_{A 21} & -\frac{D_{x c}}{D_{y}}-\alpha_{D} \frac{D_{x s}}{D_{y}} & 0 & 1
\end{array}\right) \\
& \times\left(\begin{array}{c}
\beta_{A}^{1 / 2} \epsilon_{A}^{1 / 2} \cos \mu_{A} t \\
-\alpha_{A} \beta_{A}^{-1 / 2} \epsilon_{A}^{1 / 2} \cos \mu_{A} t+\beta_{A}^{-1 / 2} \epsilon_{A}^{1 / 2} \sin \mu_{A} t \\
\beta_{D}^{1 / 2} \epsilon_{D}^{1 / 2} \cos \mu_{D} t \\
-\alpha_{D} \beta_{D}^{-1 / 2} \epsilon_{D}^{1 / 2} \cos \mu_{D} t+\beta_{D}^{-1 / 2} \epsilon_{D}^{1 / 2} \sin \mu_{D} t .
\end{array}\right) \\
= & \left(\begin{array}{c}
A_{x} \cos \mu_{A} t+D_{x c} \cos \mu_{D} t+D_{x s} \sin \mu_{D} t \\
\cdots \\
D_{y} \cos \mu_{D} t+A_{y c} \cos \mu_{A} t+A_{y s} \sin \mu_{A} t . \\
\cdots
\end{array}\right)
\end{aligned}
$$

One can check that these equations for $x_{t}$ and $y_{t}$, as well as not depending on $R_{A 21}$, are identically satisfied independent of $\alpha_{A}$ and $\alpha_{D}$, and hence cannot be used to determine those quantities. This should perhaps not be suprising for the following reason. A coordinate transformation, $x \rightarrow x, p \rightarrow p^{\prime}=p-\xi x$, transforms $M$ to

$$
M^{\prime}=\left(\begin{array}{cc}
1 & 0 \\
-\xi & 1
\end{array}\right)\left(\begin{array}{cc}
\cos \mu+\alpha \sin \mu & \beta \sin \mu \\
-\gamma \sin \mu & \cos \mu-\alpha \sin \mu
\end{array}\right)\left(\begin{array}{ll}
1 & 0 \\
\xi & 1
\end{array}\right)
$$

which is equivalent to replacing $\alpha$ by $\alpha+\xi \beta$. Since only the unmeasured "momentum" or "slope" components $p$ and $q$ are influenced by the $\alpha$ parameters, those parameters cannot be inferred by measuring only $x$ and $y$. (As stated in the introduction we are intentionally refraining from inferring slope coordinates by using adjacent BPM values.) It can be shown that the transformation (73) leaves $\mathcal{E}=\operatorname{det}|C+\bar{B}|$ (but not $e, f, g$ and $h$ ) invariant.

Should one tentatively attempt to exploit this invariance to simplify the equations by setting $\alpha_{A}=\alpha_{D}=0$ one obtains from Eq. (72), expressions for the 
unmeasured components

$$
\begin{aligned}
& p_{t}^{\prime}=\mathcal{G} \beta_{A}^{-1 / 2} \epsilon_{A}^{1 / 2} \sin \mu_{A} t+\mathcal{G} R_{A 21} \beta_{D}^{1 / 2} \epsilon_{D}^{1 / 2} \cos \mu_{D} t-\mathcal{G} \frac{A_{y c}}{A_{x}} \beta_{D}^{-1 / 2} \epsilon_{D}^{1 / 2} \sin \mu_{D} t \\
& q_{t}^{\prime}=\mathcal{G} R_{A 21} \beta_{A}^{1 / 2} \epsilon_{A}^{1 / 2} \cos \mu_{A} t-\mathcal{G} \frac{D_{x c}}{D_{y}} \beta_{A}^{-1 / 2} \epsilon_{A}^{1 / 2} \sin \mu_{A} t+\mathcal{G} \beta_{D}^{-1 / 2} \epsilon_{D}^{1 / 2} \sin \mu_{D} t
\end{aligned}
$$

where the primes indicate that $p_{t}^{\prime}$ and $q_{t}^{\prime}$ are not necessarily even approximately equal to the "correct" slope coordinates that might, for example, be obtained from processing adjacent BPM outputs. Having obtained expressions for $p_{t}^{\prime}$ and $q_{t}^{\prime}$, one can substitute into the Eqs. (46) in the forlorn hope of obtaining the asyet-undetermined quantities $\operatorname{tr} A$ and $\operatorname{tr} D$. This yields sixteen equations: 2(trig functions) times 2 (tunes) times 4 (equations). Since all these equations turn out to be satisfied identically, they cannot be used to determine any other parameters. For checking these identities one needs to use the result

$$
\frac{-\zeta}{1-\zeta} D_{y} A_{x}=A_{y c} D_{x c}+\left(\beta_{A} R_{A 21}\right) A_{y s} D_{y}
$$

It can be shown using Eqs. (24) that this is simply equivalent to the condition $\operatorname{det}\left|R_{A}\right|=\zeta /(1-\zeta)$. This can be used to determine $\zeta$ from $R_{A 21}$ or vice versa, but they are not determined independently. The expense of having checked Eqs. (46) can be charged against the QA (quality assurance) account, since self-consistency has been tested. Should we choose to trust that the global decoupling operation performed just before the data was obtained, we would substitute $\zeta=0$ to obtain

$$
\beta_{A} R_{A 21}=-\frac{A_{y c} D_{x c}}{A_{y s} D_{y}} \quad \text { (if and only if } \mathcal{E}=0 \text { ). }
$$

It seems that the best that can be done to derive the slopes $p_{t}$ and $q_{t}$ from the measurements at a single BPM is to expand the second and fourth rows of Eq. (72) using $\alpha_{x}$ and $\alpha_{y}$ in place of $\alpha_{A}$ and $\alpha_{D}$ and using Eq. (76) for $R_{A 21}$.

\section{Quantifying the Degree of Local Coupling}

The ultimate goal of our investigation is to "locally decouple" accelerator lattices. A practical algorithm for achieving this is described in the accompanying paper. Here we contemplate two candidate definitions of local coupling, justify the particular choice adopted, and supply qualitative interpretation. As part of the discussion it is necessary to define the degree of local coupling in order to be able to specify a limit that separates acceptable and unacceptable local coupling and to define a global r.m.s. average coupling. 
The angle $\theta_{A}$ defined in Eq. (45), when evaluated at local position $s$ has a clear geometric interpretation as the major axes of the ellipse that would be visible on a (non-destructive) phosphor screen placed at $s$, when only the $A$ mode is excited. In TEAPOT documentation this is known as the $A$-eigenangle. Its deviation from zero is a candidate for use in quantifying local coupling. For example, this angle might be required to be small, say less than 0.1 . However, this quantity has an undesirable feature. Because of the alternating gradient optics, the beam profile is strongly distorted as $s$ varies. At vertical focusing quads the vertical axis is stretched and the horizontal compressed. This has the effect of magnifying $\tan \theta_{A}$, proportional to the stretch ratio $\sqrt{\beta_{y} / \beta_{x}}$. The distortion is reversed at horizontally focusing quads. Requiring $\theta_{A}$ to be less than 0.1 at a vertical focusing quad location requires it to be much less than 0.1 at a horizontal focusing quad, which is probably more conservative than intended. To compensate for this effect one can define

$$
\text { normalized A-eigenangle }=\theta_{A} \sqrt{\frac{\beta_{x}}{\beta_{y}}}
$$

Plots of this quantity are shown in Fig. 10. All this discussion can be repeated for the $D$-mode. The factor $\sqrt{\beta_{x} / \beta_{y}}$ has the desired effect of moderating the variation with $s$, but it is ad hoc and the normalized eigenangle cannot be expected to behave gracefully for large eigenangles, because of its definition in terms of a transcendental function.

The decoupling algorithm in TEAPOT quantifies coupling by the quantity $\mathcal{B}^{C}(A)$, called the local coupling badness, where

$$
\sqrt{\mathcal{B}^{C}(A)}=e_{A} \sqrt{\frac{\beta_{x}}{\beta_{y}}}
$$

and $e_{A}$ is the out-of-plane/in-plane ratio defined in Eq. (43). The same discussion as in the previous paragraph can be used to motivate the inclusion of the square root factor. Because $e_{A}$ is a simple $y / x$ ratio, Eq. (78) correctly compensates the scale distortions described there. This choice is further supported by the following argument. In what might be a highly coupled lattice, consider a short sector that contains only ideal components with no skew elements. Where it enters this ideal sector the $A$-eigenmotion can be decomposed into $\left(x, x^{\prime}, y, y^{\prime}\right)$ components. Though we are describing nominally horizontal motion, it is possible for the $y$ amplitudes to be comparable to or even greater than, the $x$ amplitudes, because of strong coupling elsewhere in the lattice. Through this ideal sector these amplitudes propagate according the standard two dimensional formalism. 
In particular, the Courant-Snyder invariants $\epsilon_{x}(A)=\gamma_{x} x^{2}+2 \alpha_{x} x x^{\prime}+\overline{\beta_{x}} x^{\prime 2}$ and its $y$ counterpart $\epsilon_{y}(A)$ are conserved quantities. Realizing from Eqs. (43) that $e_{A}=y(\max ) / x(\max )$ it can be seen that $\mathcal{B}^{C}(A)$ is conserved in sectors that contain no coupling elements. Note that $\sqrt{\beta_{x} / \beta_{y}}$ rather than $\sqrt{\beta_{A} / \beta_{D}}$ is the factor that correctly gives this conservation. Plots of $\sqrt{\mathcal{B}^{C}(A)}$ are shown in Fig. 11. The angle $\sqrt{\beta_{x} / \beta_{y}} \theta_{A}$ can have either sign. When it is much less than 1 radian its magnitude is approximately equal to $\sqrt{\mathcal{B}^{C}(A)}$.

\section{Conclusions}

We consider the agreement between theory and observation to be good, simultaneously validating the data, the codes, and our understanding of the situation. The measured magic ratio data of Fig. 8 could not fit so well without the theory being essentially correct and the beam position measurement and data acquisition system yielding more or less what they were supposed to. The good agreement between two determinations of $R_{A 12}$ shown in Fig. 7 gives independent modelindependent corroboration of the inherent self-consistency of the procedures. The fact that this agreement is better when measured values $\beta_{A}$ and $\beta_{D}$ are used says that their deviations from $\beta_{x}$ and $\beta_{y}$ are meaningful and have been reliably (if not accurately) determined. Naturally the simulated data, being free of noise and distortion, gives cleaner results, but they are qualitatively similar. They are indicative of the quality of results which can, in principle, be obtained with an ideally functioning BPM system. It does not seem unreasonable to anticipate data of this general quality in the near future at LEP since all the data analysed in this paper were acquired in ten minutes without having expended much time at optimizing conditions.

This investigation was undertaken largely to anticipate the performance of beam position data acquisition designs of future colliders SSC and LHC. This investigation has shown that a system like that at LEP will reliably yield the coupling information needed for locally decoupling these large colliders. At LEP, coupling measurements are available only at every second half-cell. The SSC design calls for similar instrumentation, with $10^{4}$ turn, $x, y$ digitized pairs available for readout, on demand, at every fifth half-cell. Since cross-coupling effects are expected to be somewhat more serious for SSC than for LEP, the possibility of instrumenting every quadrupole should perhaps be considered. 

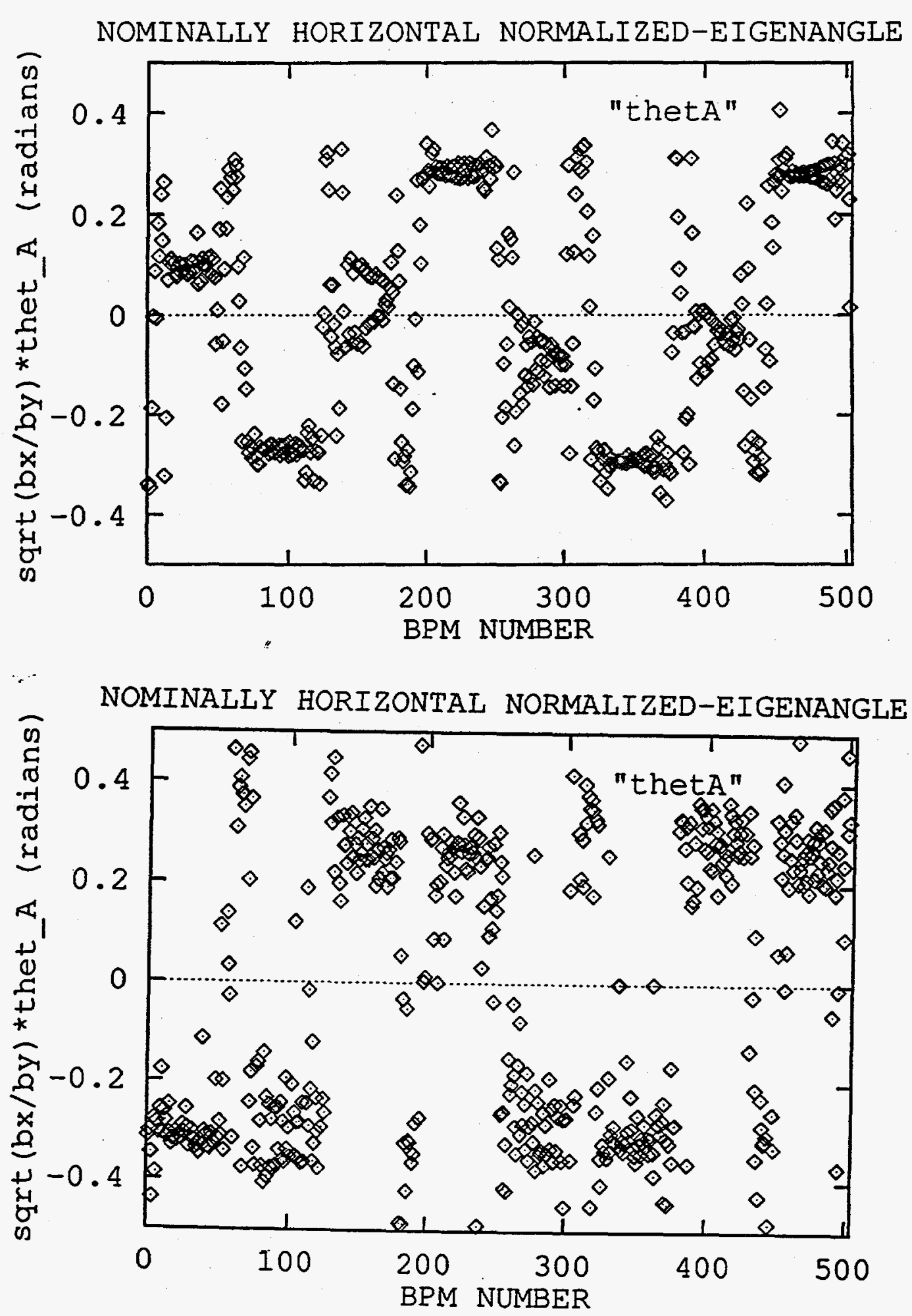

Figure 10. Normalized eigenangle $\theta_{A} \sqrt{\beta_{x} / \beta_{y}}$ evaluated at every BPM. Upper figure is from simulation, lower figure from measured data. 

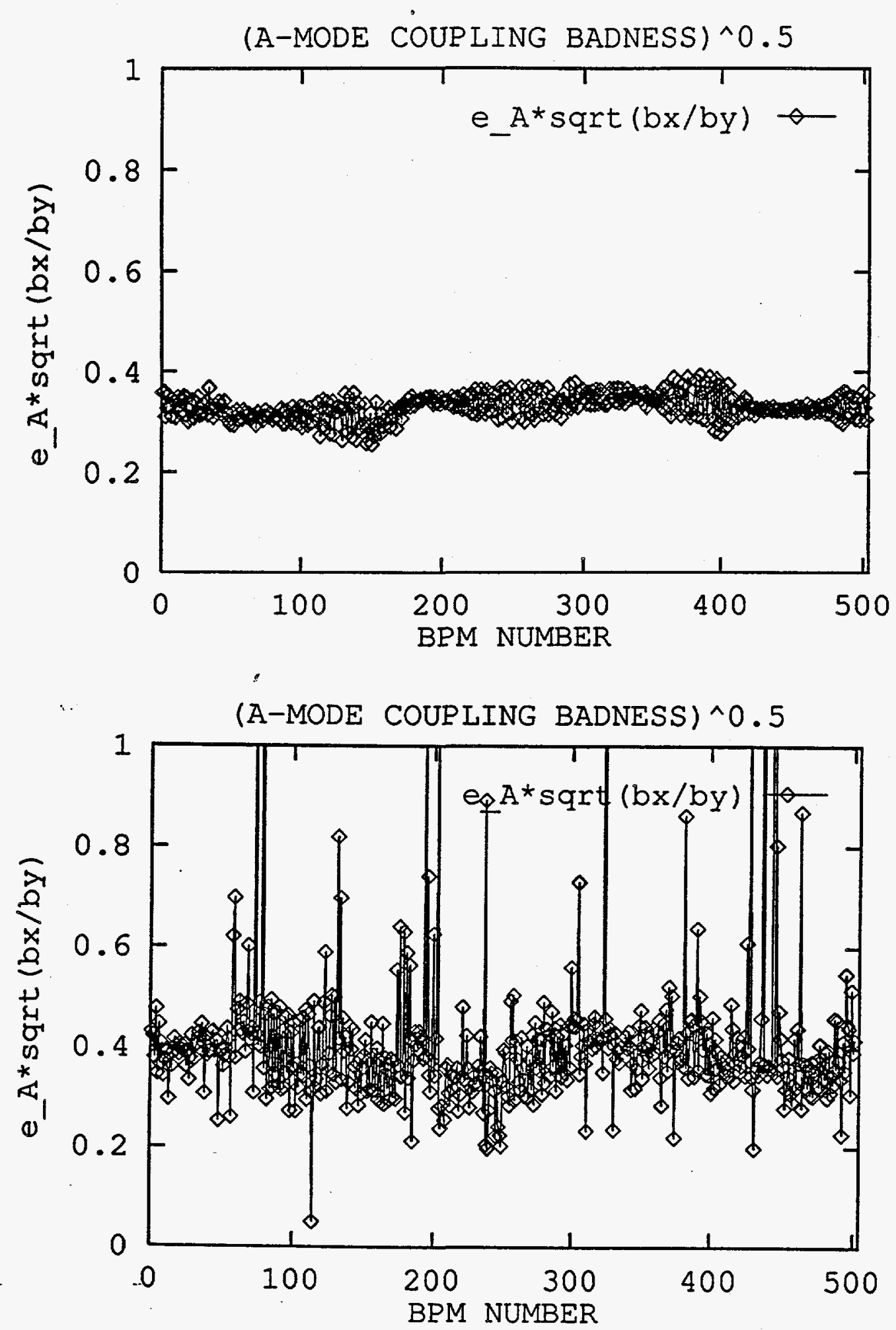

Figure 11. Square root of A-mode local coupling badness $\sqrt{\mathcal{B}^{C}(A)}=e_{A} \sqrt{\beta_{x} / \beta_{y}}$ evaluated at every BPM. Upper figure is from simulation, lower figure from measured data. 


\section{REFERENCES}

1. R. Talman, Single Particle Motion in Frontiers of Particle Beams; Observation, Diagnosis and Correction, M. Month and P. Bryant, Editors, US-CERN School on Particle Accelerators, Capri, Springer-Verlag, 1989.

2. E. Courant and H. Snyder, Annals of Physics, 3, 1 (1958).

3. L. Schachinger and R. Talman, Particle Accelerators, 22, 35 (1987). 